\title{
Two-step full waveform inversion of diving and reflected waves with the diffraction-angle-filtering-based scale- separation technique
}

\author{
Donggeon Kim ${ }^{1)}$, Jongha Hwang ${ }^{2)}$, Dong-Joo Min ${ }^{1), 3)}$, \\ Ju-Won $\mathrm{Oh}^{4)^{*}}$, and Tariq Alkhalifah ${ }^{5)}$
}

1) Seoul National University

Department of Energy Systems Engineering

1, Gwanak-ro, Gwanak-gu, Seoul 08826, Republic of Korea

2) Korea Institute of Ocean Science and Technology

Marine Active Fault Research Center

385, Haeyang-ro, Yeongdo-gu, Busan 49111, Republic of Korea

3) Seoul National University

Research Institute of Energy and Resøurces

1, Gwanak-ro, Gwanak-gu, Seoul 08826, Republic of Korea

4) Jeonbuk National University

Department of Resources and Energy Engineering

567, Baekje-daero, Deokjin-gu, Jeonju-si, Jeollabuk-do 54896, Republic of Korea

5) King Abdullah University of Science and Technology

Physical Science and Engineering Division

4700, Thuwal 23955-6900, Kingdom of Saudi Arabia

* Corresponding author

TEL: 82-63-270-2364, FAX: 82-63-270-2366, Email: juwon24@jbnu.ac.kr 
Running head: Two-step FWI with DAF-based scale-separation 


\section{SUMMARY}

Full waveform inversion (FWI) is a highly nonlinear optimization problem that aims to reconstruct high-resolution subsurface structures. The success of FWI in reflection seismology relies on appropriate updates of low-wavenumber background velocity structures, which are generally driven by the diving waves in conventional FWI. On the other hand, the reflected waves mainly contribute to updating high-wavenumber components rather than lowwavenumber components. To extract low-wavenumber information from the reflected waves in addition to the diving waves, we propose a two-step FWI strategy that separates a given model into the reflectivity and background velocity models and then alternately update them using the scale-separation technique based on diffraction-angle filtering (DAF; which was proposed to effectively control wavenumber components of the FWI gradient). Our strategy first inverts the high-wavenumber reflectivity model by suppressing energy at large diffraction angles, which are necessary to compute the reflection wavepaths (i.e., the rabbitears-shaped kernels) for low-wavenumber updates in the subsequent stage. Then, we extract low-wavenumber components due to the diving (banana-shaped kernels) and reflected waves (rabbit-ears-shaped kernels) from the gradient by suppressing energy at small diffraction angles. Our strategy is similar to reflection waveform inversion (RWI) in that it separates a given model into high- and low- wavenumber components and uses the rabbit-ears-shaped kernels for low-wavenumber updates. The main difference between our strategy and RWI is that our strategy adopts the DAF-based scale-separation technique in the space domain, which makes our algorithm of using both the banana-shaped and rabbit-ears-shaped kernels computationally attractive. By applying our two-step inversion strategy to the synthetic data from the Marmousi-II model and the real ocean-bottom cable (OBC) data from the North sea, 
we demonstrate that our method properly reconstructs low-wavenumber structures even if initial models deviate from the true models.

Keywords: Inverse theory; Waveform inversion; Seismic tomography; Body waves; Acoustic properties; Wave scattering and diffraction.

\section{INTRODUCTION}

Full waveform inversion (FWI) is a technique to provide high-resolution subsurface structures with quantitative physical properties through mathematical optimization algorithms (Tarantola 1984; Virieux and Operto 2009). However, FWI has a limitation that it is prone to fall into local minima without a kinematically accurate background velocity model (Bunks et al. 1995; Sirgue and Pratt 2004; Shen and Symes 2008; Virieux and Operto 2009). To overcome the intrinsic limitation of FWI, the low-wavenumber velocity update driven by wide-aperture seismic data is essential (Pratt et al. 1996; Brossier et al. 2015). However, in most real experiments, the data do not have long-offset components enough to recover deep structures, in which case the reconstruction of deep parts has no choice but to rely on the short-spread reflection data. Because the velocity update associated with the reflection data is dominated by high-wavenumber components (Mora 1989), conventional FWI mainly recovers short-wavelength structures for deep parts.

To extract low-wavenumber information from reflection data, reflection waveform inversion (RWI) were proposed (Xu et al. 2012) on the basis of migration-based traveltime tomography proposed by Chavent et al. (1994) and Clément et al. (2001). In RWI, low- 
wavenumber components of the gradient are computed along the reflection wavepaths (so called the rabbit-ears-shaped kernels), therefore a given velocity model should contain prior reflectivity information (Mora, 1989). RWI separates a given velocity model into the background velocity and reflectivity models and uses the reflected waves generated by the migration/demigration process to update the background velocity model. On the other hand, Zhou et al. (2015) proposed combining early-arrival waveform inversion and RWI to improve both the shallow and deep parts of the background velocity. Wu and Alkhalifah (2015) proposed a new optimization problem to simultaneously invert the background velocity and perturbation models. These RWI-based methods double the computational cost compared to conventional FWI because they are carried out in two steps of migration/demigration.

An alternative strategy to separate high- and low-wavenumber components of the gradient is to apply scattering-angle filtering using the time-lag gather, which can extract different wavenumber components of the gradient by filtering out undesired scattering-angle components (Alkhalifah 2015b). Following Alkhalifah (2015b), Wu and Alkhalifah (2017) proposed an efficient scattering-angle enrichment filtering by using the cosine function of the scattering angle as a weighting function. Yao et al. (2018) proposed a wavenumber-driven scattering-angle filtering technique, which decomposes the waves into the plane waves to easily design the opening angle filtering function. However, these scattering-angle filtering techniques would be expensive for 3D FWI because it is conducted in the wavenumber domain.

The inverse scattering imaging condition (ISIC) or energy-norm imaging condition (ENIC) (Whitmore and Crawley 2012; Ramos-Martinez et al. 2016; Rocha et al. 2016) can be considered to apply scattering-angle filtering in the space domain. ISIC or ENIC adopts the diffraction (or scattering) patterns of acoustic parameters, which represent the partial derivative wavefields with respect to model parameters. Because different parameterizations 
yield different amplitude variations of the partial derivative wavefields with respect to the scattering angles, ISIC or ENIC can control the spatial resolution of model parameter updates in FWI. Motivated from ISIC and ENIC, Oh et al. (2021) proposed the diffraction-angle filtering (DAF) technique, which provides an additional control on the intermediate scattering-angle components by imitating the virtual sources for elastic media, i.e., virtual sources for the P-wave velocity, P-wave impedance and artificially-defined Poisson's ratio.

To mitigate the intrinsic nonlinearity of FWI by preferentially utilizing low-wavenumber updates carried by both the diving and pre-/post-critical reflected waves while avoiding large increase of computational efforts, a new strategy is required. For such a method, we propose a two-step FWI strategy with the DAF-based scale-separation technique referring to the RWI framework. In our method, we split a velocity model into the high-wavenumber (reflectivity) and low-wavenumber (background velocity) scales and alternately invert them by using different modes of DAF. Through this process, we can recover low-wavenumber structures using both the banana-shaped (due to the diving waves) and rabbit-ears-shaped kernels (due to the pre-/post-critical reflected waves). Our method is an extended version of Oh et al.'s (2021) idea. Oh et al. (2021) used DAF to efficiently extract low-wavenumber components of the gradient driven mainly by the diving waves in the conventional FWI framework. However, the diving waves contained in far-offset data are vulnerable to cycle-skipping when an initial velocity model deviates from the true model, and their coverage is liable to be limited because of a limited acquisition aperture. In our strategy, DAF is applied to extract both the high- and low-wavenumber components of the gradients, which are used to update the reflectivity and background velocity models in the RWI framework. In the following sections, we first investigate the sensitivity kernels of FWI with the prior information of reflectivity, and show the performance of DAF, which enables us to easily separate the migration and tomographic components by changing the diffraction patterns with simple 
operations in the space domain. Finally, we apply our strategy to the synthetic data for the complex Marmousi-II velocity model and the real ocean-bottom cable (OBC) data from the North Sea.

\section{REVIEW OF ACOUSTIC FWI AND DIFFRACTION-ANGLE}

\section{FILTERING (DAF)}

\subsection{Formulation of acoustic FWI}

In time-domain acoustic FWI using the least-squares-norm-based local optimization, the objective function can be expressed as

$$
E(m)=\sum_{s} \sum_{r} \sum_{t} \frac{1}{2}\left[P^{c}\left(x_{s}, x_{r}, t, m\right)-P^{o}\left(x_{s}, x_{r}, t\right)\right]^{2},
$$

where $P^{o}$ and $P^{c}$ are the observed and modelled pressure data, respectively; $s, r$ and $t$ denote the source, receiver and time, respectively; and $m$ indicates the model parameter at each nodal point. The modelled data can be computed by a forward modelling algorithm satisfying the acoustic wave equation. The $2 \mathrm{D}$ acoustic wave equation with the constant density can be derived from the equation of wave motion and the generalized Hooke's law, given by

$$
\begin{gathered}
\mathbf{a}\left(x_{s}, \mathbf{x}, t, m\right)=-\nabla P\left(x_{s}, \mathbf{x}, t, m\right)+\mathbf{f}\left(x_{s}, t\right), \\
\frac{\partial^{2} P\left(x_{s}, \mathbf{x}, t, m\right)}{\partial t^{2}}=-V_{\mathrm{p}}(\mathbf{x})^{2}\left(\nabla \cdot \mathbf{a}\left(x_{s}, \mathbf{x}, t, m\right)\right)+f_{P}\left(x_{s}, t\right),
\end{gathered}
$$


where $P$ and $\mathbf{a}$ are the pressure and particle acceleration vector, respectively; $\mathbf{x}$ indicates the space coordinates; and $V_{\mathrm{p}}$ is the P-wave velocity. The terms $f_{P}$ and $\mathbf{f}$ represent the pressure and body-force sources, respectively, acting as seismic sources at the position $x_{j}$. Note that in mono-parameter acoustic case, the model parameter $m$ corresponds to the Pwave velocity (i.e., $m_{i}=V_{\mathrm{p}}\left(x_{i}\right)$ at the $i^{\text {th }}$ nodal point). By combining eqs (2) and (3), we obtain the second-order acoustic wave equation in terms of pressure written by

$$
\frac{\partial^{2} P\left(x_{s}, \mathbf{x}, t, m\right)}{\partial t^{2}}=V_{\mathrm{p}}(\mathbf{x})^{2} \nabla^{2} P\left(x_{s}, \mathbf{x}, t, m\right)+f_{P}\left(x_{s}, t\right),
$$

and the second-order acoustic wave equation in terms of particle displacement vector ( $\mathbf{u})$ given by

$$
\mathbf{a}\left(x_{s}, \mathbf{x}, t, m\right)=\nabla\left[V_{\mathrm{p}}(\mathbf{x})^{2}\left(\nabla \cdot \mathbf{u}\left(x_{s}, \mathbf{x}, t, m\right)\right)\right]+\mathbf{f}\left(x_{s}, t\right) .
$$

Because we commonly record the pressure data in marine seismie acquisition, the gradient with respect to the model parameter at the $i^{\text {th }}$ nodal point $\left(m_{i}\right)$ in acoustic FWI can be directly expressed by the zero-lag cross-correlations between the partial derivative wavefield and the pressure data residual as follows:

$$
\frac{\partial E(m)}{\partial m_{i}}=\sum_{s} \sum_{r} \sum_{t}\left[\frac{\partial P^{c}\left(x_{s}, x_{r}, t, m\right)}{\partial m_{i}} \cdot\left(P^{c}\left(x_{s}, x_{r}, t, m\right)-P^{o}\left(x_{s}, x_{r}, t\right)\right)\right] .
$$

The partial derivative pressure wavefield with respect to $m_{i}$ is computed by performing forward modelling (eq. 4) using the virtual pressure source $f_{m_{i}, s}^{v}$ (Pratt et al. 1998), which can be derived by differentiating eq. (4) with respect to $m_{i}$. To avoid huge computational overburden arising from the direct calculation of the partial derivative wavefields for all the nodal points, the gradient with respect to $m_{i}$ is computed using the adjoint-state method (Plessix 2006) as follows: 


$$
\frac{\partial E(m)}{\partial m_{i}}=\sum_{s} \sum_{r} \sum_{t}\left[f_{m_{i}, s}^{v}\left(x_{i}, t, m\right) \cdot P_{s}^{b}\left(x_{r}, x_{i}, T-t, m\right)\right]
$$

where

$$
f_{m_{i}, s}^{v}\left(x_{i}, t, m\right)=2 V_{\mathrm{p}}\left(x_{i}\right) \nabla^{2} P\left(x_{s}, x_{i}, t, m\right) \text {. }
$$

The term $P_{s}^{b}$ is the adjoint pressure wavefield computed by back-propagating the pressure data residuals between $P^{c}$ and $P^{o}$.

The gradient (eq. 7) can also be reformulated in terms of the virtual body-force source and the back-propagated adjoint particle acceleration wavefield. By taking partial derivatives of eqs (2) and (3) with respect to $m_{i}$ and assuming the equivalent body-force source instead of the pressure source, the partial derivative pressure wavefield can be expressed by

$$
\begin{gathered}
\frac{\partial \mathbf{a}^{c}\left(x_{s}, \mathbf{x}, t, m\right)}{\partial m_{i}}=-\nabla\left(\frac{\partial P^{c}\left(x_{s}, \mathbf{x}, t, m\right)}{\partial m_{i}}\right)+\mathbf{f}_{m_{i}, s}^{v}\left(x_{i}, t, m\right) \\
\frac{\partial^{2}}{\partial t^{2}}\left(\frac{\partial P^{c}\left(x_{s}, \mathbf{x}, t, m\right)}{\partial m_{i}}\right)=-V_{p}(\mathbf{x})^{2}\left(\nabla \cdot\left(\frac{\partial \mathbf{a}^{c}\left(x_{s}, \mathbf{x}, t, m\right)}{\partial m_{i}}\right)\right) .
\end{gathered}
$$

Combining eqs (9) and (10), we can obtain the second-order acoustic wave equation for the partial derivative pressure wavefield with the equivalent virtual body-force source as follows:

$$
\begin{gathered}
\frac{\partial^{2}}{\partial t^{2}}\left(\frac{\partial P^{c}\left(x_{s}, \mathbf{x}, t, m\right)}{\partial m_{i}}\right)=V_{p}(\mathbf{x})^{2} \nabla^{2}\left(\frac{\partial P^{c}\left(x_{s}, \mathbf{x}, t, m\right)}{\partial m_{i}}\right)+f_{m_{i}, s}^{v}\left(x_{i}, t, m\right), \\
f_{m_{i}, s}^{v}\left(x_{i}, t, m\right)=-V_{p}(\mathbf{x})^{2} \nabla \cdot \mathbf{f}_{m_{i}, s}^{v}\left(x_{i}, t, m\right) .
\end{gathered}
$$

The virtual body-force source $\mathbf{f}_{m_{i}, s}^{v}$ of eq.(12) can be computed by differentiating eq. (5) with respect to $m_{i}$ following the definition of the virtual source given by Pratt et al. (1998) as below:

$$
\mathbf{f}_{m_{i}, s}^{v}\left(x_{i}, t, m\right)=\nabla\left[2 V_{\mathrm{p}}\left(x_{i}\right) \nabla \cdot \mathbf{u}\left(x_{s}, x_{i}, t, m\right)\right]
$$

Based on vector identities, the adjoint method and eq. (12), the gradient of eq. (7) can be 
rewritten (Oh et al. 2021) by

$$
\frac{\partial E(m)}{\partial m_{i}}=\sum_{s} \sum_{t}\left[\mathbf{f}_{m_{i}, s}^{v}\left(x_{i}, t, m\right) \cdot \mathbf{a}_{s}^{b}\left(x_{r}, x_{i}, T-t, m\right)\right]
$$

where

$$
\mathbf{a}_{s}^{b}\left(x_{r}, \mathbf{x}, T-t, m\right)=V_{p}(\mathbf{x})^{2} \nabla P_{s}^{b}\left(x_{r}, \mathbf{x}, T-t, m\right) .
$$

Note that the gradient can be computed in terms of either the virtual pressure source (eq. 7 ) or virtual body-force source (eq. 14). When we use eq. (14), the particle acceleration wavefield is numerically obtained using the finite-difference formula of eq. (2). Therefore, the acoustic FWI gradient in terms of the virtual body-force source can be computed from the secondorder acoustic wave equation in terms of the pressure (eq. 4) without a significant increase in the computational cost. Based on eqs (13) - (15), Oh et al. (2021) modified the virtual source for acoustic FWI to imitate the P-P diffraction patterns for the P-wave/velocity and P-wave impedance in elastic FWI with the $V_{\mathrm{p}}-I_{\mathrm{p}}-\sigma$ parameterization, where $V_{\mathrm{p}}, I_{\mathrm{p}}$ and $\sigma$ are the Pwave velocity, P-wave impedance and Poisson's ratio, respectively (Oh and Min, 2017). The details will be discussed in Section 2.3.

\subsection{Sensitivity kernel of acoustic EWI}

To examine the nonlinear property of acoustic FWI for reflection-dominated data, we compute the sensitivity kernel for a 1D velocity model with a single reflector assuming a pair of source and receiver (Fig. 1). In Fig. 1(a), we observe that the sensitivity kernel for the smoothed model without any reflectors is composed of the low-wavenumber first Fresnel zone (i.e., the banana-shaped kernel) and the high-wavenumber migration isochrone (Woodward 1992; Virieux and Operto 2009). Because there are no reflectors in the beginning, only the down-going forward and adjoint wavefields exist. The down-going adjoint wavefield 
consists of two components: One is from the diving-wave residual, and the other is from the reflected-wave residual. While the former contributes to building of the first Fresnel zone, the latter to the generation of the migration isochrone. Stacking the migration isochrones inverts the reflector. Once the reflector is recovered, both the forward and adjoint wavefields generate up-going components. The cross-correlation between the up-going component of the forward wavefield and down-going component of the adjoint wavefield of the reflected-wave residual builds the first Fresnel zone. Likewise, the down-going component of the forward wavefield and up-going component of the adjoint wavefield of the reflected-wave residual produce another first Fresnel zone (Xu et al. 2012; Brossier et al. 2015). These first Fresnel zones appear in a rabbit-ears shape in Fig. 1(b). Because the banana-shaped kernel cannot reach the deep parts without a long-spread acquisition geometry, the rabbit-ears-shaped kernel is mainly in charge of the recovery of low-wavenumber structures of the deep parts. As we see in Fig. 1(b), the migration isochrone (whose strength is proportional to reflection coefficient $R$ ) is much stronger than the rabbit-ears-shaped kernel (whose strength is proportional to $R^{2}$ ) (Zhan et al. 2014). For this reason, in conventional FWI, lowwavenumber structures of the deep parts are not properly recovered, but high-wavenumber reflectors are dominantly reconstructed by the migration isochrones (Mora 1989). This coupling of high- and low-wavenumber components of the gradient can cause strong nonlinearity in case initial models are not accurate enough to image reflectors at their correct positions. To mitigate the nonlinearity of conventional FWI, techniques of separating the high- and low-wavenumber components of the gradient have been used.

\subsection{A review of DAF}

To efficiently separate high- and low-wavenumber components of the gradient, we 
introduce the DAF technique (Oh et al. 2021). The main idea of DAF is to modify the virtual source term to control the diffraction patterns of the partial derivative wavefields. Based on the expression of the virtual body-force source represented in eq. (13), DAF modifies the conventional virtual source of the P-wave velocity as follows (Oh et al. 2021):

$$
\begin{aligned}
\mathbf{f}_{m_{i}, s}^{v, \mathrm{DAF}}\left(x_{i}, t, m\right)= & a_{1} \nabla\left[V_{p}\left(x_{i}\right) \nabla \cdot \mathbf{u}\left(x_{s}, x_{i}, t, m\right)\right]+a_{2}\left[\frac{1}{V_{p}\left(x_{i}\right)} \mathbf{a}\left(x_{s}, x_{i}, t, m\right)\right], \\
& +a_{1}\left(\frac{1-2 \sigma}{2-2 \sigma}\right)\left[\frac{\partial \mathbf{s}\left(x_{s}, \mathbf{x}, t, m\right)}{\partial m_{i}}\right]
\end{aligned}
$$

where

$$
\begin{aligned}
\frac{\partial \mathbf{s}\left(x_{s}, \mathbf{x}, t, m\right)}{\partial m_{i}}= & -2 \nabla\left(V_{p}\left(x_{i}\right)\right)\left(\nabla \cdot \mathbf{u}\left(x_{s}, x_{i}, t, m\right)\right) \\
& +\nabla\left(V_{p}\left(x_{i}\right)\right) \cdot\left[\nabla \mathbf{u}\left(x_{s}, x_{i}, t, m\right)+\left(\nabla \mathbf{u}\left(x_{s}, x_{i}, t, m\right)\right)^{T}\right] . \\
& -V_{p}\left(x_{i}\right)\left[\nabla \times\left(\nabla \times \mathbf{u}\left(x_{s}, x_{i}, t, m\right)\right)\right]
\end{aligned}
$$

The last term in eq. (17) is zero in acoustic cases, because the curl of the P-wave is zero. In eq. (16), there are three weighting factors (represented by $a_{1}, a_{2}$ and artificially-defined Poisson's ratio $\sigma$ ), which control the influences of the three terms (i.e., three square brackets). The first term is the conventional virtual source of the P-wave velocity as shown in eq. (13). The second term controls energy at either small or large diffraction angles as ISIC or ENIC does. The last term additionally suppresses energy at intermediate diffraction angles, which can cause the leakage of high-wavenumber components of gradient during tomographic FWI. The second and last terms can be activated by changing a set of weighting factors $\left(a_{1}, a_{2}\right.$ and $\sigma$ ) as shown in Table 1 . The virtual source obtained applying mode I is identical to the conventional virtual source of the P-wave velocity. The virtual sources resulting from applying modes II and III are the same as the virtual sources of the P-wave velocity and Pwave impedance obtained from the acoustic wave equation with the $V_{\mathrm{p}}-I_{\mathrm{p}}$ parameterization, 
respectively. Modes IV and V describe the virtual sources identical to those of the P-wave velocity and $\mathrm{P}$-wave impedance in the elastic equations with the $V_{\mathrm{p}}-I_{\mathrm{p}}-\sigma$ parameterization, respectively. We set artificial Poisson's ratio $(\sigma)$ to be 0.25 to activate the last term without generating artificial backscattering energy (Oh et al. 2021).

Fig. 2 shows the diffraction patterns generated by the virtual sources resulting from applying the five DAF modes. Mode I yields an isotropic diffraction pattern (Fig. 2a). The diffraction patterns of mode II (Fig. 2b) and mode III (Fig. 2c) diffract energy over intermediate diffraction angles, which causes cross-talk effects between high- and lowwavenumber components of the gradient. On the other hand, mode IV (Fig. 2d) and mode V (Fig. 2e) play a role in suppressing the diffraction energy at intermediate diffraction angles by mimicking the diffraction patterns of the P-wave velocity and P-wave impedance in elastic FWI with $V_{\mathrm{p}}-I_{\mathrm{p}}-\sigma$ parameterization. Compared with the previously-proposed scattering-angle filtering methods, DAF controls the diffraction pattern by modifying the virtual source in the space domain. Because no transforms are required across the data domains, DAF can be easily applied to a large-scale problem like 3D FWI. While scattering-angle filtering suggested by Alkhalifah (2015b) can design a more sophisticated filter, modes IV and V of DAF can effectively separate the migration and tomographic components with lower computational efforts. 


\section{TWO-STEP FWI STRATEGY}

Oh et al. (2021) designed DAF and only applied mode IV of DAF to recover lowwavenumber structures in the conventional FWI framework. In this case, low-wavenumber information is mainly extracted from the diving and post-critical reflected waves (Zhou et al. 2015), and thus tends to be confined to relatively shallow depths depending on the acquisition geometry. In addition, the diving waves contained in far-offset data are vulnerable to cycle skipping. As a result, to successfully recover subsurface structures using Oh et al.'s (2021) strategy, we need long-offset data and a good initial guess. However, the data acquísition geometry is often limited for various reasons, in which case low-wavenumber information for deeper parts should be extracted from the pre-critical reflected waves. The low-wavenumber information driven from short-spread reflection data can be relatively robust to cycle skipping. To recover low-wavenumber structures over the whole subsurface model using both the diving and pre-/post-critical reflected waves, we propose a two-step FWI strategy with the DAF-based scale-separation technique in the RWI framework.

\subsection{Sensitivity kernel filtered by DAF}

We investigate characteristics of the sensitivity kernels filtered by DAF. Fig. 3 shows the filtered versions of the sensitivity kernels displayed in Fig. 1. The black lines indicate the reflector $\delta m$. In Figs $3($ a) $-(\mathrm{d})$, we observe that the sensitivity kernels of FWI are dominated by the high-wavenumber migration isochrones, which indicates that modes III and V sufficiently suppress the low-wavenumber first Fresnel zones related with the diving (indicated by the blue arrows in Figs $3 \mathrm{a}$ and $\mathrm{c}$ ) and reflected waves (indicated by the green 
arrows in Figs $3 b$ and d), respectively. In addition, the parts of migration isochrones at large diffraction angles (indicated by the yellow arrows) have been suppressed compared with those at small diffraction angles (indicated by the red arrows), which implies that the migration isochrones at near offsets will be boosted with modes III or V of DAF. In contrast, the sensitivity kernels filtered by modes II (Figs 3e and f) and IV (Figs 3g and h) show different aspects. Because mode II does not suppress energy at intermediate diffraction angles, the migration isochrone in Fig. 3(e) is not sufficiently attenuated (indicated by the red and yellow arrows). In Fig. 3(f), amplitudes of the migration isochrone are still comparable with those of the rabbit-ears-shaped kernel, which makes it difficult to focus on inverting the background velocity. In contrast, after filtered by mode IV, the migration isochrone is well suppressed, and thus the kernel is dominated by the low-wavenumber first Fresnel zone related to the diving wave (indicated by the blue arrow in Fig. 3g). Likewise, the sensitivity kernel in Fig. 3(h) is dominated by the low-wavenumber banana-shaped and rabbit-earsshaped kernels (indicated by the blue and green arrows). However, the parts of migration isochrones at large diffraction angles remain to some extent (indicated by the yellow arrows) compared with those with small diffraction angles (indicated by the red arrows), which implies that the migration isochrones at far offsets still contribute to updating the velocity structures even with mode II or IV of DAF. In the conventional FWI framework, the kernels in Fig. 3(g) contribute to recovering low-wavenumber structures with mode IV of DAF. In contrast, in two-step FWI, the gradient is carried by the kernels in Fig. 3(h). The bananashaped kernels or migration isochrones at far offsets mainly recover the low-verticalwavenumber structures along the horizontal wavepaths and requires long-offset components, while the rabbit-ears-shaped kernels recover the low-horizontal-wavenumber structures along the vertical wavepaths and can be utilized with relatively short offsets (Mora 1989; Alkhalifah 2015a; Zhou et al. 2018). In such a complementary manner, the two-step FWI can 
provide wider low-wavenumber coverage.

\subsection{Design of two-step FWI with the DAF-based scale-separation technique}

The two-step FWI strategy separates a given velocity model $(m)$ into a small-scale highwavenumber reflectivity model representing reflectivity $(\delta m)$ and a large-scale lowwavenumber background velocity model $\left(m_{0}\right)$. Then, we alternately invert the two separated models. Our algorithm has some similarities with RWI (Xu et al. 2012) or the nested inversion algorithm (Biondi and Almomin 2014; Wang et al. 2016). The main difference is that we directly separate high-wavenumber and low-wavenumber components from the full gradient by using modes $\mathrm{V}$ and IV of DAF, respectively. Compared with computationally expensive preprocessing techniques (e.g., the migration/demigration, or up-/down-going wavefield separation, etc.), our strategy only requires simple additional operations to solve the spatial derivatives of wavefields for DAF (Oh et al. 2021).

The former studies for RWI showed that $\delta m$ should be inverted by fitting the zerooffset data to preserve the zero-offset traveltimes and subsequently match traveltimes at far offsets by inverting $m_{0}$ to avoid local minima problem (Brossier et al. 2015; Zhou et al. 2015; Wang et al. 2016; Chen et al. 2020). Because mode V of DAF extracts the migration isochrones at small diffraction angles as mentioned in Section 3.1, it can be useful in inverting $\delta m$ by fitting the near-zero-offset reflection data. In contrast, because mode IV of DAF suppresses the migration isochrones and extracts the low-wavenumber kernels, it can be used for reconstruction of $m_{0}$. Therefore, the new gradient directions for the high- and the 


$$
\frac{\partial E\left(m_{0}, \delta m\right)}{\partial \delta m_{i}}=\sum_{s} \sum_{r} \sum_{t}\left[\mathbf{f}_{m_{i}, s}^{v, \text { Mode v }}\left(x_{i}, t, m_{0}, \delta m\right) \cdot \mathbf{a}_{s}^{b}\left(x_{r}, x_{i}, T-t, m_{0}, \delta m\right)\right],
$$

and

$$
\frac{\partial E\left(m_{0}, \delta m\right)}{\partial m_{0, i}}=\sum_{s} \sum_{r} \sum_{t}\left[\mathbf{f}_{m_{i}, s}^{v, \text { Mode IV }}\left(x_{i}, t, m_{0}, \delta m\right) \cdot \mathbf{a}_{s}^{b}\left(x_{r}, x_{i}, T-t, m_{0}, \delta m\right)\right],
$$

where the superscripts "Mode IV" and "Mode V" indicate the virtual sources filtered by modes IV and V, respectively. Note that the only difference between eqs (18) and (19) is the virtual source, which controls the diffraction patterns of the partial derivative wavefields as shown in Fig. 2.

\subsection{Workflow of two-step FWI}

Considering that the low-wavenumber components along the reflection wavepaths require the prior high-wavenumber reflectivity model $\delta m$, we propose a two-step FWI workflow consists of one inner loop and one outer loop as shown in Table 2. Assuming a smooth initial background velocity $m_{0}$, our workflow begins with the inner loop where $\delta m$ is updated for reflectivity inversion. In the inner loop, by using mode V of DAF (eq. 18), we mainly utilize the near-normal-incidence data. As mode V of DAF suppresses the first Fresnel zones associated with the direct/diving waves, we do not have to apply any data-separation techniques to remove the diving waves from the data. After $\delta m$ converges to some extent after several iterations, we compute the update direction for $m_{0}$ by applying mode IV of DAF (eq. 19) to the gradient corresponding to the velocity model $m_{0}+\delta m$. In other words, we smoothly update the background model $m_{0}$ by extracting both banana-shaped and rabbitears-shaped kernels with mode IV of DAF. With the newly updated background velocity 
model, we return to the inner loop to re-invert $\delta m$ so that the zero-offset traveltimes of reflected waves in the newly updated background velocity model can be consistent (Brossier et al. 2015; Zhou et al. 2018). In this manner, we iterate this two-step process until $m_{0}$ converges to the true background velocity.

\section{APPLICATION TO SYNTHETIC DATA: MARMOUSI-II MODEL}

\subsection{Modelling and inversion parameters}

To assess the performance of our two-step FWI algorithm, we apply it to the synthetic pressure data computed for the Marmousi-II model shown in Fig.4(a) (Martin et al. 2006; several interfaces are indicated by the black arrows). The model is discretized by $460 \times 153$ grid points with a grid interval of $20 \mathrm{~m}$. We assume that 46 shots and 460 receivers are applied with an even interval at a depth of $20 \mathrm{~m}$, and that the shot and receiver intervals are 200 and $20 \mathrm{~m}$, respectively. We use a linearly-increasing 1-D velocity model for an initial model as shown in Fig. 4(b). For the source function, we use a Ricker wavelet with a peak frequency of $6.5 \mathrm{~Hz}$. The total recording length is $5.2 \mathrm{~s}$. We apply the zero-phase Butterworth filters with bandwidths of $3-6,3-9$ and $3-12 \mathrm{~Hz}$ to the observed and modelled data for the multiscale strategy (Bunks et al. 1995). For numerical modelling, we use the 4th-order staggered-grid finite-difference method (Virieux 1984) and convolutional perfectly matched layers (Komatitsch and Martin 2007) are added to avoid artificial boundary reflections. Fig. 5 shows a representative shot gather at a distance of $1.9 \mathrm{~km}$. We can observe the pre-critical reflected waves at near-to-intermediate offsets (in the red box) and the diving and postcritical reflected waves at far offsets (outside the red box). In the workflow described in Table 
2, we invert reflectivity model using near-offset data in the inner loop, and in the outer loop, background velocity model is recovered using the full-offset data. The preconditioned steepest-descent method is applied for the inner and outer loops. The gradient is preconditioned by the pseudo-Hessian matrix (Shin et al. 2001). Considering that incompletely inverted reflectivity boosts the high-wavenumber artefacts and hinders the contribution of the rabbit-ears-shaped kernels to the update of the background velocity model, we iterate the inner loop by 10 times for each background velocity model so that the amplitudes and phases of reflected waves can be well matched with those of the observed data at near offsets. We also iterate the outer loop by 10 times for each frequency band. We use a fixed step-length of $0.05 \mathrm{~km} / \mathrm{s}$ for both the inner and outer loops.

\subsection{Inversion results}

As mentioned in Section 3.3, after inverting $\delta m$ for the initially given background model $m_{0}$ in the inner loop, the update direction for $m_{0}$ is computed by applying mode IV of DAF to the gradient corresponding to the velocity model $m_{0}+\delta m$ in the outer loop (Table 2). Fig. 6 shows the resultant model $m_{0}+\delta m$ after 10 iterations of the inner loop. In Fig. 6, we observe that some reflectors are mispositioned (pointed by the black arrows), which can be moved to the correct positions by using additional low-wavenumber information carried by the rabbit-ears-shaped kernels. Recall that mode V of DAF suppresses the low-wavenumber components of the gradient and boosts the contribution of the nearnormal-incidence data. Fig. 7 shows filtered versions of the gradients computed at the first iteration of the outer loop. To examine if the pre-critical reflected waves can provide complementary low-wavenumber coverage for the background velocity, which cannot be 
covered by only the diving waves, we separate the pre-critical reflection data and earlyarrival data (diving and post-critical reflected waves) by using a linear time-offset window (the red box in Fig. 5). To demonstrate if energy at intermediate diffraction angles is suppressed by mode IV of DAF, the gradients filtered by mode IV are compared to those filtered by mode II (whose mechanism is identical to the ENIC proposed by Rocha et al. 2016). Because mode IV of DAF applied in the outer loop additionally suppresses the energy at intermediate diffraction angles, the gradient filtered by mode IV in Fig. 7(b) reveals macrostructures of the velocity model without high-wavenumber artefacts compared with that filtered by mode II in Fig. 7(a). The gradients obtained using only the early-arrival data are shown in Figs 7(c) and (d). In Figs 7(c) and (d), the gradients mainly carried by the bananashaped kernels reveal the low-wavenumber structures up to a depth of $2.5 \mathrm{~km}$ along the paths of diving waves. However, due to the limitation of acquisition geometry, the left-hand and right-hand sides of the model are hardly delineated. Likewise, mode IV shows better performance compared with mode II. In contrast, the gradients obtained using only the precritical reflection data provide the information of macro-structures at distances of $0-3 \mathrm{~km}$ and $7-9 \mathrm{~km}$ in Figs 7(e) and (f). Because strong reflectors are located at depths of $2-2.5$ $\mathrm{km}$, the gradients in Figs 7(e) and (f) mainly delineate the structures above a depth of $2 \mathrm{~km}$. The migration isochrones at intermediate diffraction angles are dominant in this case and thus high-wavenumber artefacts seyerely occur in the gradient filtered by mode II compared with that filtered by mode IV, which shows the performance of mode IV in dealing with precritical reflection data. In Fig. 8, we present the background velocity models corresponding to the gradients in Fig. 7. Figs 8(a) and (b) demonstrate that our two-step FWI using both the banana-shaped and rabbit-ears-shaped kernels yields the best low-wavenumber coverage, which reflects macro-structures of the Marmousi-II model, compared with Figs 8(c) - (f). In 
Figs $8(\mathrm{c})$ and (d), while the shallow parts of the background velocity are improved by the contribution of the diving waves, the left-hand side of the model (at distances of $0-3 \mathrm{~km}$ ) and macro-structures at depths of $1-2 \mathrm{~km}$ are not recovered well even though the diving waves can reach the deepest part of the model. This is probably due to the limited wavenumber coverage of the banana-shaped kernels as mentioned in Section 3.1 and cycle skipping of far-offset data containing the diving waves. When an initial model deviates from the true model, far-offset data required to retrieve low-wavenumber information of the deep parts are prone to be cycle-skipped, which causes the local minima problem. On the other hand, Figs 8(e) and (f) show that two-step FWI strategy using the pre-critical reflection data resolves macro-structures different from those delineated by two-step FWI strategy using the early-arrival data, specifically above a depth of $2 \mathrm{~km}$, which leads to wider coverage of lowwavenumber structures in Figs 8(a) and (b). Note that the background velocity models obtained by two-step FWI filtered by mode II (Figs 8a, c and e) contain some highwavenumber artefacts and do not fully resolve macro-structures compared with those filtered by mode IV (Figs 8b, d and f).

To compare our results with those of Oh et al. (2021), we display the gradient at the first iteration and the final model obtained by applying mode IV of DAF in conventional FWI in Figs 9 and 10. By comparing Fig. 9 with Fig. 7, we see that the gradient of Fig. 9 looks similar to that of Fig. 7(d), which indicates that mode IV of DAF plays a role in recovering low-wavenumber information carried by the banana-shaped kernels in the conventional FWI framework. Accordingly, the resultant background velocity in Fig. 10 cannot recover reliable macro-structures as in the inverted background velocity in Fig. 8(d). From these results, we can confirm that our two-step FWI strategy boosts up the contribution of the rabbit-earsshaped kernels, which provides complementary low-wavenumber information.

The background velocity model can be used as an initial model in subsequent FWI 
performed to recover detailed velocity structures. For subsequent FWI, we apply the conventional FWI algorithm. Figs 11(a) and (b) show the final velocity models inverted by subsequent FWI starting from the linearly-increasing velocity model (i.e., initial model) and the background velocity model recovered by our two-step FWI strategy, respectively. When we start from the linearly-increasing model, we fail to recover the accurate velocity model because of the severe traveltime errors due to the inaccurate initial model (Fig. 11a). In contrast, when we start from the background velocity reconstructed by our two-step FWI strategy, we obtain inversion results comparable to the true velocity model (Fig. 11b). In Fig. 12, we show absolute differences between the inverted models and the true velocity model. For reference, we also display the difference between the linearly-increasing and true velocity models. In Fig. 12, we observe that the differences for the case of starting from the background velocity obtained by our strategy (Fig. 12b) are smaller than those for both the linearly-increasing velocity model (Fig. 12c) and the final model inverted starting from the linearly-increasing velocity model (Fig. 12a), specifically at depths of $0-2 \mathrm{~km}$. Fig. 13(a) shows shot gathers of the observed and modelled data generated for the finally inverted model (e.g., Fig. 11b), which indicate that the amplitudes and phases of refractions and reflections are also well-recovered. Fig. 13(b) also supports that traces of the observed and modelled data match well with each other. These results convince us that the success of FWI depends on the recovery of reliable background velocity, which can be accomplished by effectively extracting low-wavenumber information from both the diving and pre-/postcritical reflected waves. 


\section{APPLICATION TO FIELD DATA: VOLVE OBC DATA}

\subsection{Modelling and inversion parameters}

We examine the performance and applicability of our two-step FWI algorithm for field data by applying it to the 3D OBC data acquired in the Volve oil field of the North Sea in 2002 (Szydlik et al. 2007). For 2D acoustic inversion, we choose a single cable fine containing 240 receivers with an interval of $25 \mathrm{~m}$. The preprocessed data have officially been released by Equinor and its former Volve partners since October 2018. We additionally apply a simple procedure of $3 \mathrm{D}$-to-2D conversion to compensate for the amplitude and phase errors caused by 2D approximation (Crase et al. 1990). The dimension of model is $12 \mathrm{~km} \times 4.5$ $\mathrm{km}$ with a grid spacing of $25 \mathrm{~m}$. 60 pressure sources were applied with an interval of $200 \mathrm{~m}$ at a depth of $25 \mathrm{~m}$, and 240 receivers were deployed at the sea bottom ranging from 3.175 to $9.15 \mathrm{~km}$ of the model. Although the water depth varies from 80 to $100 \mathrm{~m}$, we assume a flat sea bottom with a water depth of $100 \mathrm{~m}$. Because inversion is mainly performed with lowfrequency data, the depth variation is less than one grid point. The recording length is $6.4 \mathrm{~s}$. For stable FWI, we apply the zero-phase Butterworth filter with corner frequencies of 2.5 and $5.6 \mathrm{~Hz}$ to the data.

For forward modelling, we use the same technique that was used in Section 4. A representative shot gather and its band-pass filtered version are shown in Figs 14(a) and (b), respectively. For reference, we display the 3D tomography model released along with the data in Fig. 15(a). The black solid lines indicate the path of the well and the yellow dashed lines indicate the 2D line extracted for acoustic inversion. For an initial velocity model, we use a linearly-increasing model as shown in Fig. 15(b). As we did for the Marmousi-II model, 
we iterate the inner loop of Table 2 by 10 times for each background velocity model to invert the reflectivity model. The total iteration number for the outer loop is 12 . For simplicity, we use a fixed step length of $0.05 \mathrm{~km} / \mathrm{s}$ for both the inner and outer loops.

To minimize amplitude mismatches caused by inaccurate physical approximations (i.e., acoustic approximation of elastic media) and geometrical spreading effects remaining after 3D-to-2D conversion, we use the least-squares norm with trace-by-trace-normalized data (i.e., global correlation norm) as an objective function (Shen 2010; Choi and Alkhalifah 2012; Warner et al. 2013). We estimate the source wavelet by matching near-offset direct arrivals of the trace-by-trace-normalized observed and modelled data using the filter-based method (Pratt, 1999). Compared to the synthetic example, the Volve/data have weak reflections as shown in Fig. 14. Therefore, we scale the gradient with the depth-variable weighting factor $\left(e^{2 z}\right.$, where $z$ indicates the depth) to amplify the deeper parts in both the inner and outer loops of Table 2. As addressed in the former studies, there are strong anisotropic effects at depths of $1-2.5 \mathrm{~km}$ in the Volve oil field (Oh et al. 2018; Oh and Alkhalifah 2019; Li and Alkhalifah 2020). According to Zhang and Alkhalifah (2017) and Feng and Schuster (2019), we do not use the far-offset data to mitigate anisotropic effects. The maximum offset was adjusted from $9 \mathrm{~km}$ to $3 \mathrm{~km}$. Then, the gradient is mainly affected by the rabbit-ears-shaped kernels driven from the near-to-intermediate-offset data, which is relatively free from trade-off effects due to the anisotropic parameters.

\subsection{Inversion results}

As we did for the synthetic data in Section 4, we begin by inverting the highwavenumber reflectivity $\delta m$ for the initial background velocity $m_{0}$ shown in Fig. 15(b). 
Fig. 16 shows the resultant model $m_{0}+\delta m$ obtained by applying mode V of DAF after 10 iterations of the inner loop. We observe that the horizontal chalk layer is inverted indicating the location of reservoir, as in the previous studies (Szydlik et al. 2007; Guo and Alkhalifah 2017; Oh et al. 2018; Li et al. 2019). This horizontal reflector will play a role in providing the reflection wavepaths at depths of $0-3 \mathrm{~km}$ during inversion of the background velocity model in the outer loop. The background velocity is updated by applying mode IV of DAF to the gradient corresponding to the velocity model $m_{0}+\delta m$. Figs 17 and 18 show the scaled gradient and the background velocity model obtained by our two-step FWI strategy, respectively. In Fig. 17, the gradient contains the low-wavenumber components at depths of $1.5-3 \mathrm{~km}$, which are carried by the rabbit-ears-shaped kernels associated with the precritical reflected waves. Even though we only use the near-to-intermediate-offset data, we were able to update the low-wavenumber structures at the deeper depths of $1.5-3 \mathrm{~km}$. Fig. 18 shows the background velocity model after 12 iterations of the outer loop, where we can also confirm that macro-structures at depths shallower than $3 \mathrm{~km}$ are well recovered. This low-wavenumber update will play a role in locating the chalk layer in its correct position.

We subsequently carry out conventional FWI for the Volve data using the background velocity model recovered by our two-step FWI strategy (Fig. 18) as an initial guess. For comparison, we also perform FWI starting from the linearly-increasing velocity model (Fig. 15b). Fig 19 shows the final inversion results and the corresponding depth profiles along the well path starting from the linearly-increasing velocity model and the background velocity model obtained by two-step FWI. The well is oblique and deviates from the 2D line for inversion. Therefore, we project the well path along the 2D line. The prominent reflector due to horizontal chalk interface in the left panel of Fig. 19(a) is located at depths deeper than 3 $\mathrm{km}$ and not focused well. In the right panel of Fig. 19(a), we observe that the linearly- 
increasing velocity model (represented by the green line) is far from the well log (black) and the final inversion result starting from the linearly-increasing velocity model (red) deviates from the tendency of the well log and does not match well with the P-wave velocity contrast due to chalk interface. In contrast, the reflector in the left panel of Fig. 19(b) is shifted to a shallower depth which is presumably its correct position (refer to the black arrows that indicates the top of the chalk layer in tomography model). In the right panel of Fig. 19(b), we observe that the background velocity model inverted by two-step FWI (green) has lower velocities than those of the linearly-increasing velocity model (green in Fig. 19a). Accordingly, the final inversion result starting from the background velocity (red) matches well with the tendency of the well log compared with that starting from the linearlyincreasing velocity model (red in Fig. 19a), specifically above a depth of $2.3 \mathrm{~km}$. The upper boundary of the cap rock is shifted up to some extent. Still, the locations of the cap rock and reservoir are slightly different from the well log. Considering that the depth of the chalk layer varies from 2.5 to $3 \mathrm{~km}$ along the inline or crossline directions, which forms a small domeshaped structure (Szydlik et al. 2007; refer to the reference 3D tomography model in Fig. 15a), these mismatches might be caused by the errors due to the projection of the oblique well deviated from our 2D line.

For another quality control, angle-domain common-image gathers (ADCIGs; Sava and Fomel 2003) are computed for the final FWI results obtained starting from the linearlyincreasing velocity model and with the background velocity model inverted by two-step FWI, which are displayed ín Figs 20(a) and (b), respectively. The events in Fig. 20(b) are much flatter than those of Fig. 20(a). As strong reflections generated by the chalk layer mainly contribute to low-wavenumber update like RWI, the events at depths of $2.5-3 \mathrm{~km}$ (in the yellow dashed box in Fig. 20) are flattened well. However, because we mainly recovered the vertical P-wave velocity, some events at large angles still smile upward, which might be 
attributed to anisotropy (indicated by the red arrows). In Fig. 21, we compare shot gathers of the trace-by-trace-normalized observed and the modelled data for the finally inverted model (e.g., Fig. 19b). In Fig. 21, we observe that the phases of refractions and reflections are wellmatched at the near-to-intermediate offsets (shorter than $4-5 \mathrm{~km}$ ). From these results, we confirm that the reconstructed P-wave velocity model reasonably describes the observed pressure data acquired in the Volve oil field.

\section{DISCUSSION}

In acoustic FWI for field data, the inherent anisotropic and elastic properties of subsurface media might degrade the quality of inversion results. Because of the inaccurate approximations of physical behaviors of seismic waves in real seismic survey, the amplitudes of field and modelled data show significantly different aspects. Meanwhile, the traveltime differences caused by the anisotropic effects in the Volve oil field become remarkable at offsets longer than $4 \mathrm{~km}$ (Oh et a1. 2018). The previous studies of acoustic inversion for VTI media (Zhang and Alkhalifah 2017; Feng and Schuster 2019) have showed that the trade-off effects between P-wave velocity and anisotropic parameter $\varepsilon$ are severe at large opening angles, which implies that the responses due to the P-wave velocity and $\varepsilon$ are not distinguished in the low-wavenumber kernels driven by far-offset data. Because Oh et al. (2021) only used the low-wavenumber information driven by the diving and post-critical reflected waves at far offsets employing mode IV of DAF, the horizontal P-wave velocity was mainly recovered at the upper layers with strong anisotropy. In contrast, our strategy recovers 
the vertical P-wave velocity using the rabbit-ears-shaped kernels, which is less affected by anisotropy. Meanwhile, mono-parameter acoustic inversion cannot fully recover large contrasts of the P- and S-wave impedances (e.g., at the chalk interface) due to amplitude mismatches caused by the $\mathrm{P}-\mathrm{S}$ mode conversion (Agudo et al. 2020). For further improvements, we need to perform elastic FWI to simultaneously recover the P-wave and Swave structures by using the inverted P-wave background velocity model as an initial guess.

\section{CONCLUSIONS}

We proposed the two-step FWI strategy with the DAF-based scale-separation technique that can provide wider low-wavenumber coverage for the reconstruction of the background velocity model. By separating the high- and low-wavenumber components of the gradient with mode $\mathrm{V}$ and IV of DAF and alternately inverting them, we can combine the bananashaped and rabbit-ears-shaped kernels for the recovery of low-wavenumber background velocity structures while suppressing the high-wavenumber migration components. The synthetic data example on the Marmousi-II model illustrated that the proposed two-step FWI strategy can build a reasonable background velocity model even without prior knowledge of subsurface structures. The inversion results obtained by our two-step FWI strategy indicate that jointly using diving and reflected waves is critical to properly recover the lowwavenumber information over the entire subsurface model. The strategy was also applied to the field data set from the North Sea. A high-resolution velocity model was obtained by first recovering the background velocity using the two-step FWI strategy and applying subsequent 
FWI. Our two-step FWI strategy can be used to provide reliable background P-wave velocity models that can be used as initial guesses for further multi-parameter inversion for elastic VTI media. We also expect that our strategy for low-wavenumber updates can also be efficiently applied for 3D cases.

\section{ACKNOWLEDGEMENTS}

This research was supported by the National Research Foundation of Korea (NRF) funded by the Ministry of Science and ICT (NRF-2019K1A3A1A80113341; NRF2020R111A3073977), and the Human Resources Development program (No.20204010600250) of the Korea Institute of Energy Technology Evaluation and Planning (KETEP) funded by the Ministry of Trade, Industry, and Energy of the Korean Government. Research reported in this publication was also supported by competitive research funding from the King Abdullah University of Science and Technology (KAUST) in Thuwal, Saudi Arabia. Also, the North sea data are released by Equinor and former Volve license Partners under Creative Commons License. We greatly appreciate their efforts to disclose the Volve data. The views on the Volve data expressed in this paper are the views of the authors and do not necessarily reflect the views of Equinor and former Volve license Partners. Lastly, we appreciate the editor and anonymous reviewers for their careful reading of our manuscript and their insightful comments and suggestions. 


\section{DATA AVAILABILITY}

The North sea data are released by Equinor and former Volve license Partners under Creative Commons License. The code underlying this article will be shared on reasonable request to the corresponding author. 


\section{REFERENCES}

Agudo, Ò.C., da Silva, N.V., Stronge, G. \& Warner, M., 2020. Mitigating elastic effects in marine 3-D full-waveform inversion, Geophys. J. Int., 220, 2089-2104.

Alkhalifah, T., 2015a. Conditioning the full waveform inversion gradient to welcome anisotropy, Geophysics, 80, R111-R122.

Alkhalifah, T., 2015b. Scattering-angle based filtering of the waveform inversion gradients, Geophys. J. Int., 200(1), 363-373.

Biondi, B. \& Almomin, A., 2014. Simultaneous inversion of full data bandwidth by tomographic full-waveform inversion, Geophysics, 79(3), WA129-WA140.

Brossier, R., Operto, S. \& Virieux, J., 2015. Velocity model building from seismic reflection data by full waveform inversion, Geophys. Prospect., 63, 354-367.

Bunks, C., Saleck, F.M., Zaleski, S. \& Chavent, G., 1995. Multiscale seismic waveform inversion, Geophysics, 60(5), 1457-1473.

Chavent, G., Clément, F. \& Gómez, S., 1994. Automatic determination of velocities via migration-based traveltime waveform inversion: a synthetic data example, SEG Technical Program Expanded Abstracts 1994, pp. 1179-1182.

Chen, Y., Feng, Z., Fu, L., AlTheyab, A., Feng, S. \& Schuster, G., 2020. Multiscale reflection phase inversion with migration deconvolution, Geophysics, 85(1), R55-R73.

Choi, Y. \& Alkhahifah, T., 2012. Application of multi-source waveform inversion to marine streamer data using the global correlation norm, Geophys. Prospect., 60(4), 748-758.

Clément,F., Chavent, G. \& Gómez, S., 2001. Migration-based traveltime waveform inversion 2 of 2-D simple structures: a synthetic example, Geophysics, 66, 845-860.

Crase, E., Pica, A., Noble, M., McDonald, J. \& Tarantola, A., 1990. Robust elastic non-linear 
waveform inversion: application to real data, Geophysics, 55, 527-538.

Feng, S. \& Schuster, G. T., 2019. Transmission + reflection anisotropic wave-equation traveltime and waveform inversion, Geophys. Prospect., 67, 423-442.

Guo, Q. \& Alkhalifah, T., 2017. Elastic reflection-based waveform inversion with a nonlinear approach, Geophysics, 82(6), R309-R321.

Komatitsch, D. \& Martin, R., 2007. An unsplit convolutional Perfectly Matched Layer improved at grazing incidence for the seismic wave equation, Geophysics, 72(5), SM155-SM167.

Li, Y., Guo, Q., Li, Z. \& Alkhalifah, T., 2019. Elastic reflection waveform inversion with variable density, Geophysics, 84(4), R553-R567.

Li, Y. \& Alkhalifah, T., 2020. Multi-parameter reflection waveform inversion for acoustic transversely isotropic media with a vertical symmetry axis, Geophys. Prospect., 68, $1878-1892$.

Martin, G. S., Wiley, R. \& Marfurt, K. J., 2006. Marmousi2: An elastic upgrade for Marmousi, Leading Edge, 25, 156-166.

Mora, P.R., 1989. Inversion = migration + tomography, Geophysics, 54(12), 1575-1586.

Oh, J.-W. \& Min, D.-J., 2017. Multi-parameter full waveform inversion using Poisson's ratio for elastic media, Explor. Geophys., 48(4), 456-475.

Oh, J.-W., Kalita, M. \& Alkhahifah, T., 2018. 3D elastic full-waveform inversion using Pwave excitation amplitude: Application to ocean bottom cable field data, Geophysics, 83(2), R129-R140.

Oh, J.-W. \& Alkhalifah, T., 2019. Study on the full-waveform inversion strategy for 3D elastic orthorhombic anisotropic media: application to ocean bottom cable data, Geophys.

Prospect., 67, 1219-1242.

Oh, J.-W., Cheng, J. \& Min, D.-J., 2021. Diffraction-angle filtering of gradient for acoustic 
full-waveform inversion, Geophysics, 86(2), R173-R185.

Plessix, R.-E., 2006. A review of the adjoint-state method for computing the gradient of a functional with geophysical applications, Geophys. J. Int., 167(2), 495-503.

Pratt, R.G., Song, Z.-M., Williamson, P. \& Warner, M., 1996. Two-dimensional velocity models from wide-angle seismic data by wavefield inversion, Geophys. J. Int., 124, $323-340$.

Pratt, R.G., Shin, C. \& Hick, G., 1998. Gauss-Newton and full newton methods in frequency space seismic waveform inversion, Geophys. J. Int., 133(2), 341-362.

Pratt, R.G., 1999. Seismic waveform inversion in the frequency domain, Part 1: Theory and verification in a physical scale model, Geophysics, 64(3), 888-901.

Ramos-Martinez, J., Crawly, S., Zou, Z., Valenciano, A.A., Qiu, L. \& Chemingui, N., 2016. A robust gradient for long wavelength FWI updates, in 78th Conference and Exhibition, EAGE, Extended Abstracts.

Rocha, D., Tanushev, N. \& Sava, P., 2016. Acoustic wavefield imaging using the energy norm, Geophysics, 81(4), S151-S163.

Sava, P.C. \& Fomel, S., 2003. Angle-domain common-image gathers by wavefield continuation methods, Geophysics, 68(3), 1065-1074.

Shen, P. \& Symes, W. W., 2008. Automatic velocity analysis via shot profile migration, Geophysics, 73(5), VE49-VE59.

Shen, X., 2010. Near-surface velocity estimation by weighted early-arrival waveform inversion, SEG Technical Program Expanded Abstracts 2010, pp. 1975-1979.

Shin, C., Jang, S. \& Min, D.-J., 2001. Improved amplitude preservation for prestack depth migration by inverse scattering theory, Geophys. Prospect., 49(5), 592-606.

Sirgue, L. \& Pratt, R.G., 2004. Efficient waveform inversion and imaging: A strategy for selecting temporal frequencies, Geophysics, 69(1), 231-248. 
Szydlik, T., Smith, P., Way, S., Aamodt, L. \& Friedrich, C., 2007. 3D PP/PS prestack depth migration on the Volve field, First Break, 25, 43-47.

Tarantola, A., 1984. Inversion of seismic reflection data in the acoustic approximation, Geophysics, 49(8), 1259-1266.

Virieux, J., 1984. SH-wave propagation in heterogeneous media: Velocity-stress finitedifference method, Geophysics, 49(11), 1933-1942.

Virieux, J. \& Operto, S., 2009. An overview of full-waveform inversion in exploration geophysics, Geophysics, 74(6), WCC1-WCC26.

Wang, F., Donno, D., Chauris, H., Calandra, H. \& Audebert, F., 2016. Waveform inversion based on wavefield decomposition, Geophysics, 81(6), R457-R470.

Warner, M., Ratcliffe, A., Nangoo, T., Morgan, J., Umpleby, A., Shah, N., Vinje, V., Štekl, I., Guasch, L., Win, C., Conroy, G. \& Bertrand, A., 2013. Anisotropic 3D full-waveform inversion, Geophysics, 78(2), R59-R80.

Whitmore, N.D. \& Crawley, S., 2012. Applications of RTM inverse scattering imaging conditions, in SEG Technical Program Expanded Abstracts 2012, pp. 1-6.

Woodward, M.J., 1992. Wave-equation tomography, Geophysics, 57, 15-26.

Wu, Z. \& Alkhalifah, T., 2015. Simultaneous inversion of the background velocity and the perturbation in full-waveform inversion, Geophysics, 80(6), R317-R329.

Wu, Z. \& Alkhalifah, T., 2017 Efficient scattering-angle enrichment for a nonlinear inversion of the background and perturbations components of a velocity model, Geophys. J. Int., 210, 1981-1992.

Xu, S., Wang, D., Chen, F., Lambare, G. \& Zhang, Y., 2012. Inversion on reflected seismic wave, in 82nd Ann. Internat. Mtg., pp. 1-7, Soc. Expl. Geophys.

Yao, G., da Silva, N. V., Warner, M. \& Kalinicheva, T., 2018. Separation of migration and tomography modes of full-waveform inversion in the plane wave domain, J. Geophys. 
Res. Solid Earth, 123, 1486-1501.

Zhan, G., Dai, W., Zhou, M., Luo, Y. \& Schuster, G. T., 2014. Generalized diffraction-stack migration and filtering of coherent noise, Geophys. Prospect., 62, 427-442.

Zhang, Z.-d. \& Alkhalifah, T., 2017. Full waveform inversion using orented time-domain imaging method for vertical transverse isotropic media, Geophys. Prospect., 65, 166180.

Zhou, W., Brossier, R., Operto, S. \& Virieux, J., 2015. Full waveform inversion of diving and reflected waves for velocity model building with impedance inversion based on scale separation, Geophys. J. Int., 202(3), 1535-1554.

Zhou, W., Brossier, R., Operto, S., Virieux, J. \& Yang, P., 2018. Velocity model building by waveform inversion of early arrivals and reflections: A 2D case study with gas-cloud effects, Geophysics, 83(2), R141-R157. 
Table 1. Five DAF modes and their weighting factors

\begin{tabular}{lc}
\hline DAF mode & Weighting factors $\left(a_{1}, a_{2}\right.$ and $\left.\sigma\right)$ \\
\hline Mode I & $(2,0,0.5)$ \\
Mode II & $(1,1,0.5)$ \\
Mode III & $(1,1,0.5)$ \\
Mode IV & $(1,-1,0.25)$ \\
Mode V & \\
\hline
\end{tabular}


Table 2. Workflow of two-step FWI with DAF

Input: Observed data $P_{o}$, initial background velocity model $m_{0}$

Outer loop: Solve $\min m_{0} E\left(m_{0}, \delta m\right)$

Inner loop: Solve $\min \delta m \quad E\left(m_{0}, \delta m\right)$

Use velocity $m_{0}$

Get gradient $\partial E\left(m_{0}, \delta m\right) / \partial \delta m_{i}$ with mode $\mathrm{V}$ of DAF

Update $\delta m$

\section{End inner loop}

Use velocity $m_{0}+\delta m$

Get gradient $\partial E\left(m_{0}, \delta m\right) / \partial m_{0, i}$ with mode IV of DAF

Update $m_{0}$

Subtract $\delta m$ from $m_{0}+\delta m$ for next inner loop

\section{End outer loop}

Output: Updated background velocity $m_{0}$ 

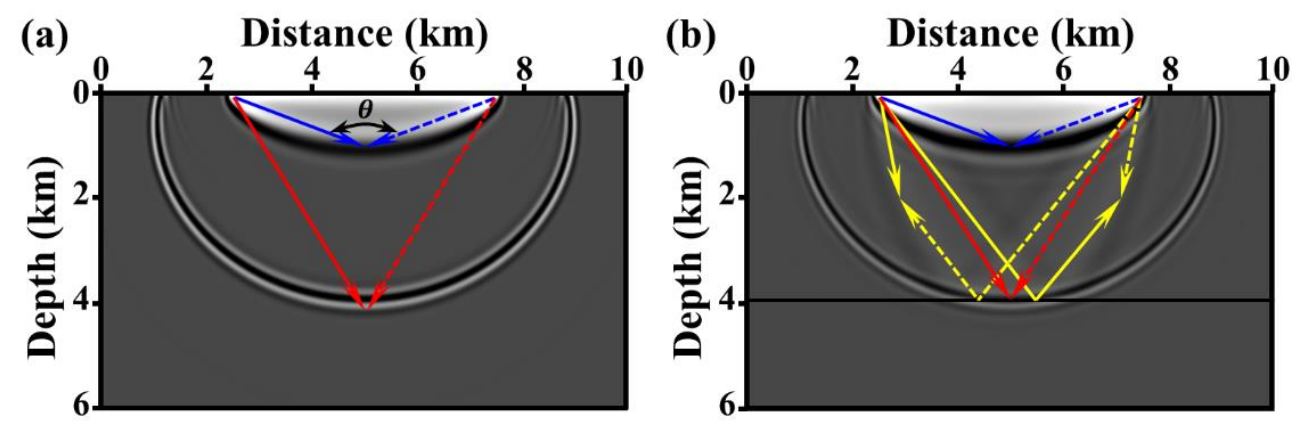

Fig. 1. The sensitivity kernels of acoustic FWI for one source-receiver pair in initial models (a) without and (b) with the prior reflector. The black line indicates the prior reflector. The solid and dashed arrows denote the ray paths of the forward and adjoint wavefields, respectively. $\theta$ is the aperture angle between the two wavefields. The cross-correlation of the blue arrows forms the first Fresnel zone associated with the diving-wave residual, while the cross-correlation of the red arrows forms the migration isochrone associated with the reflected-wave residual. With the prior reflector, the cross-correlations of the yellow arrows generate a pair of the first Fresnel zones associated with the reflected-wave residual. 

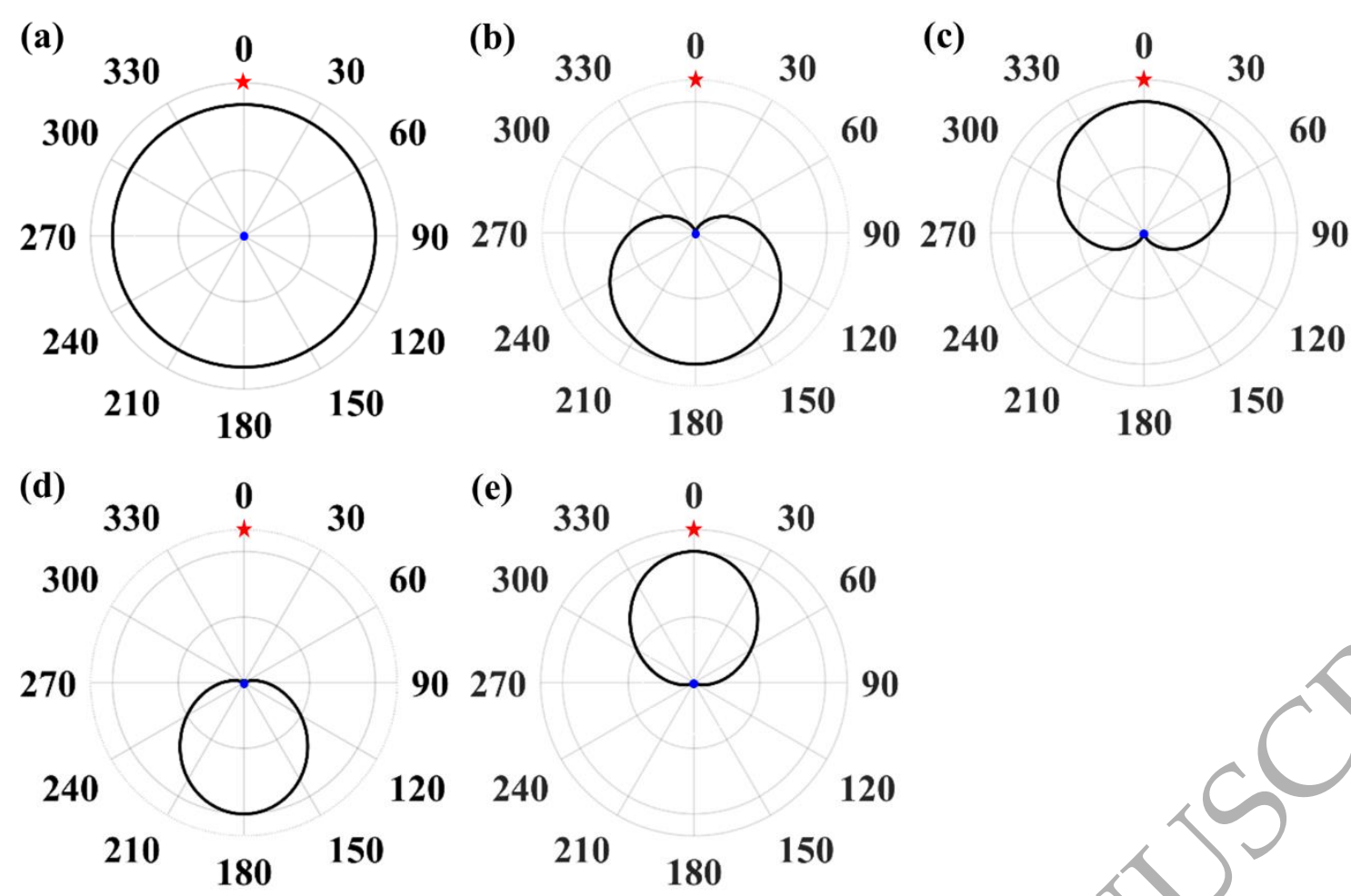

Fig. 2. Diffraction patterns of the P-wave velocity filtered by (a) mode I, (b) mode II, (c) mode III, (d) mode IV, and (e) mode V. The red stars and blue dots indicate the location of seismic source and virtual source of the P-wave velocity, respectively. 

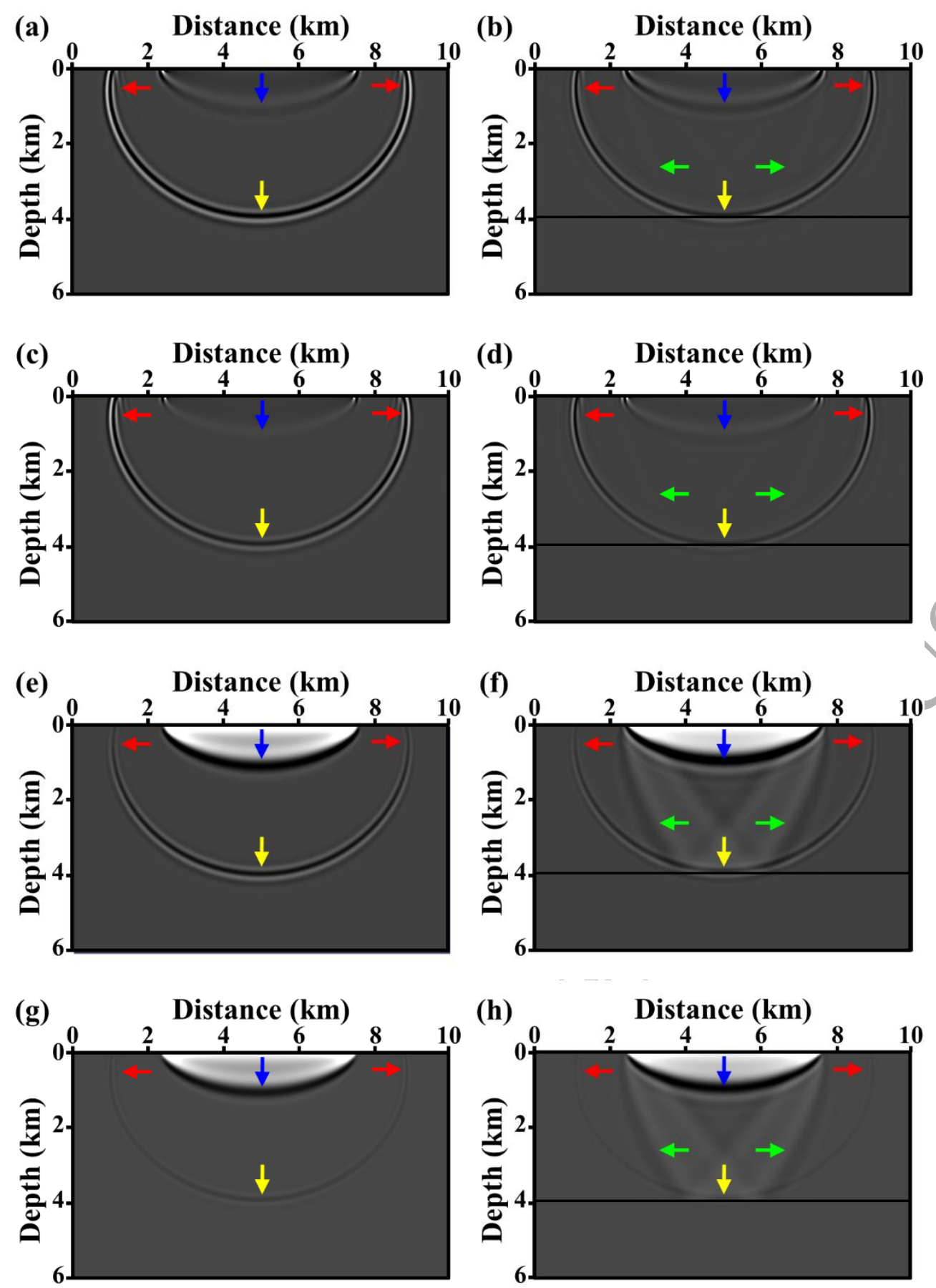

Fig. 3. The sensitivity kernels for one source-receiver pair in initial models without/with the prior reflector, filtered by $(\mathrm{a}, \mathrm{b})$ mode III, $(\mathrm{c}, \mathrm{d})$ mode $\mathrm{V},(\mathrm{e}, \mathrm{f})$ mode II and $(\mathrm{g}, \mathrm{h})$ mode IV of DAF. The black lines indicate the inverted prior reflector $\delta m$. 

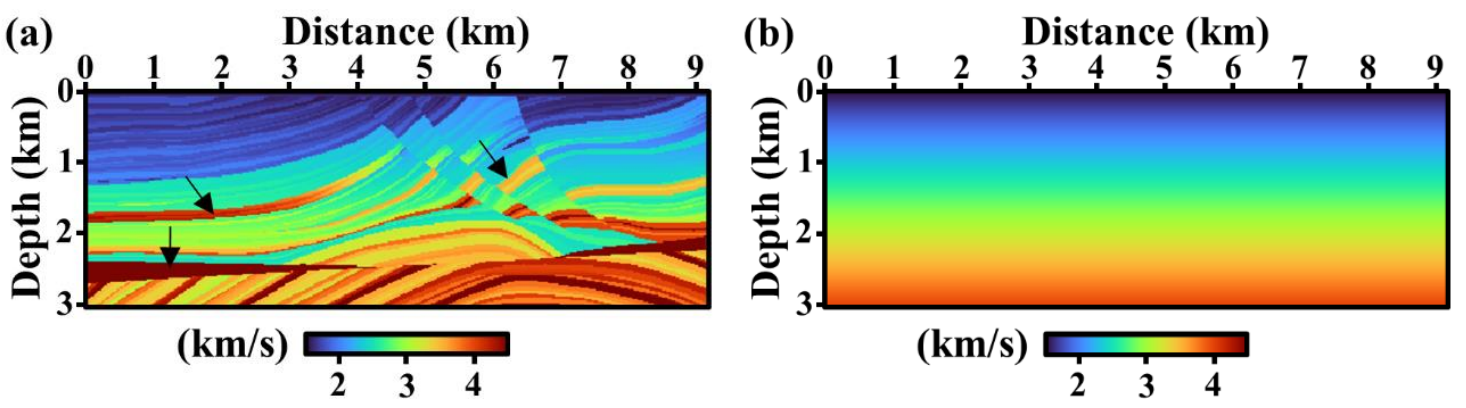

Fig. 4. (a) The true Marmousi-II velocity model and (b) the linearly-increasing velocity model used as an initial guess. The black arrows will be used for comparison with Fig. 11. 


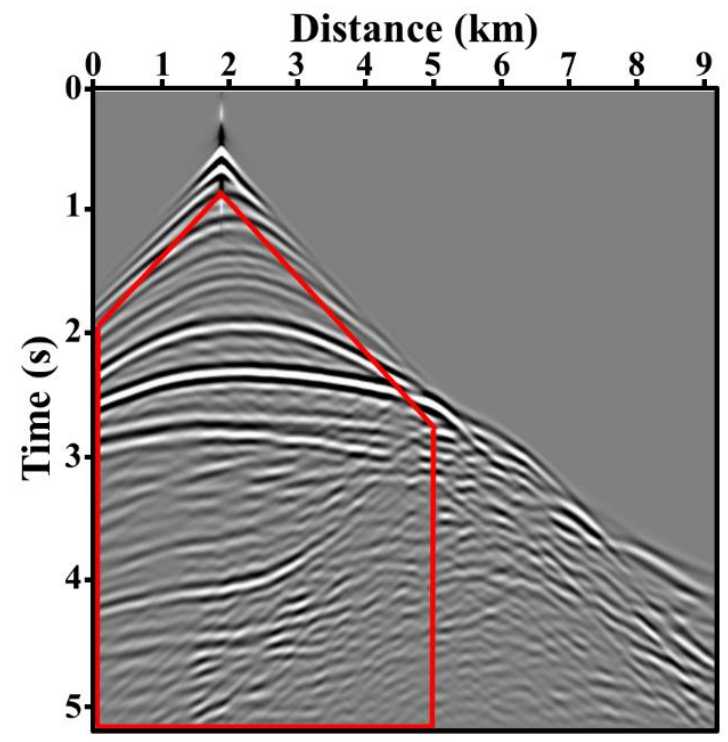

Fig. 5. A representative shot gather at a distance of $1.9 \mathrm{~km}$ of the Marmousi-II model. The seismic data contain the pre-critical reflected (in the red box), diving and post-critical reflected waves (outside the red box). 


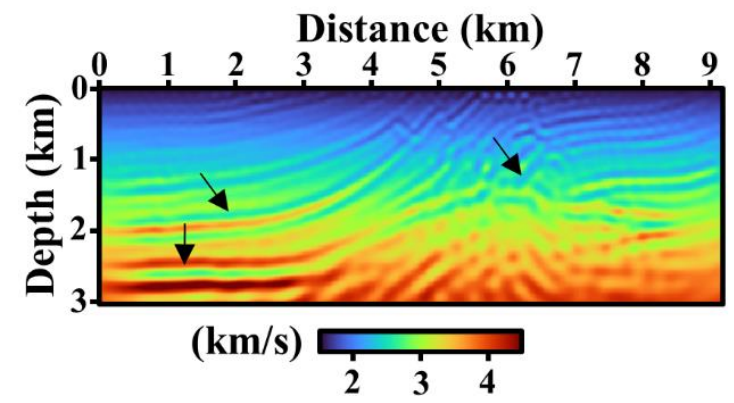

Fig. 6. The high-wavenumber velocity model $m_{0}+\delta m$ inverted by using mode $\mathrm{V}$ of DAF in the inner loop of two-step FWI, when the background velocity model $m_{0}$ is assumed to be the linearly-increasing velocity model shown in Fig. 4(b). 

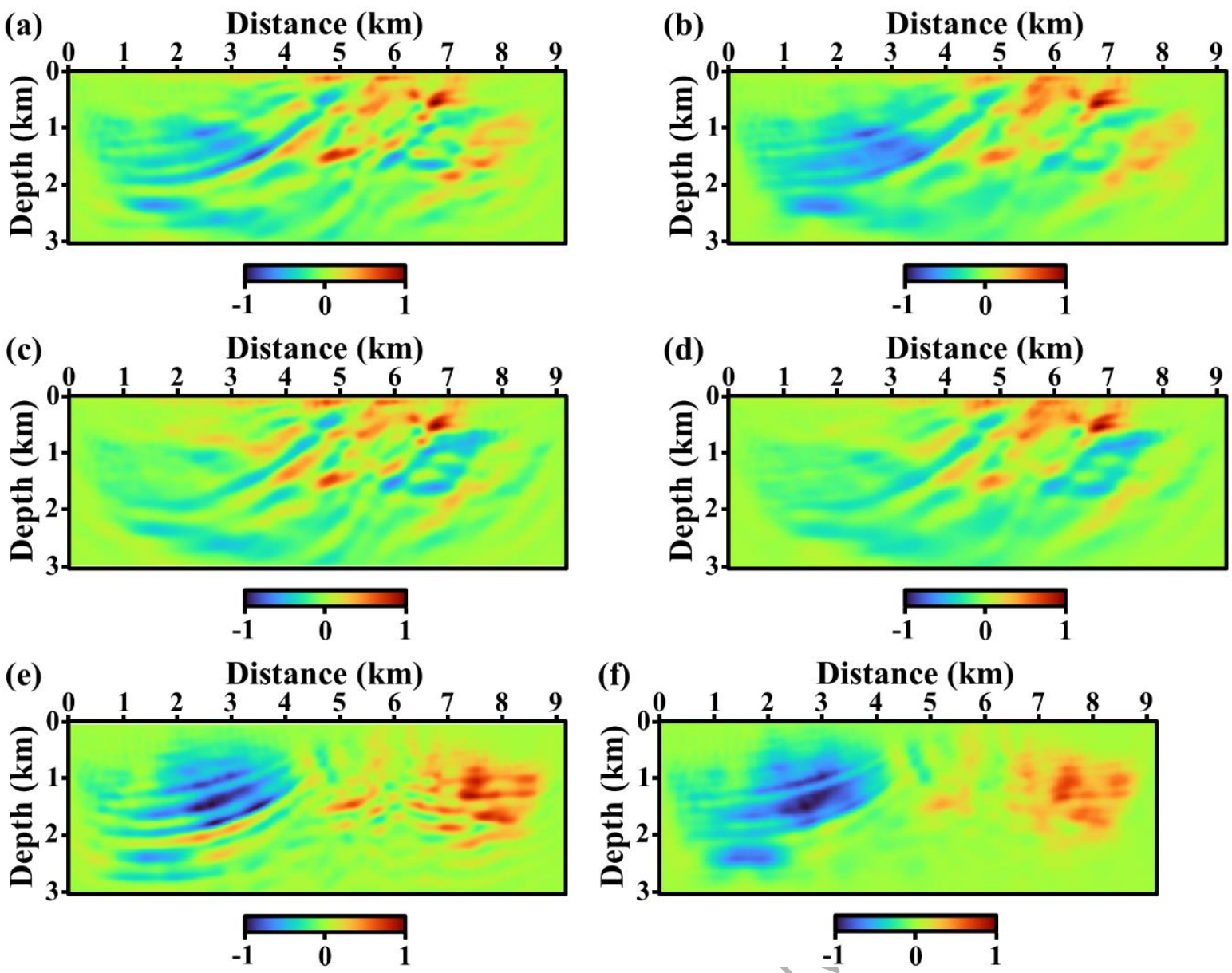

Fig. 7. The gradients obtained at the first iteration of the outer loop of two-step FWI using (a, b) the full data, (c, d) the early arrival data (diving and post-critical reflected waves; outside the red box in Fig. 5) and (e, f) the pre-critical reflection data (in the red box in Fig. 5). The left panels (a, c, e) are filtered by mode II of DAF and the right panels (b, d, e) are filtered by mode IV of DAF. 

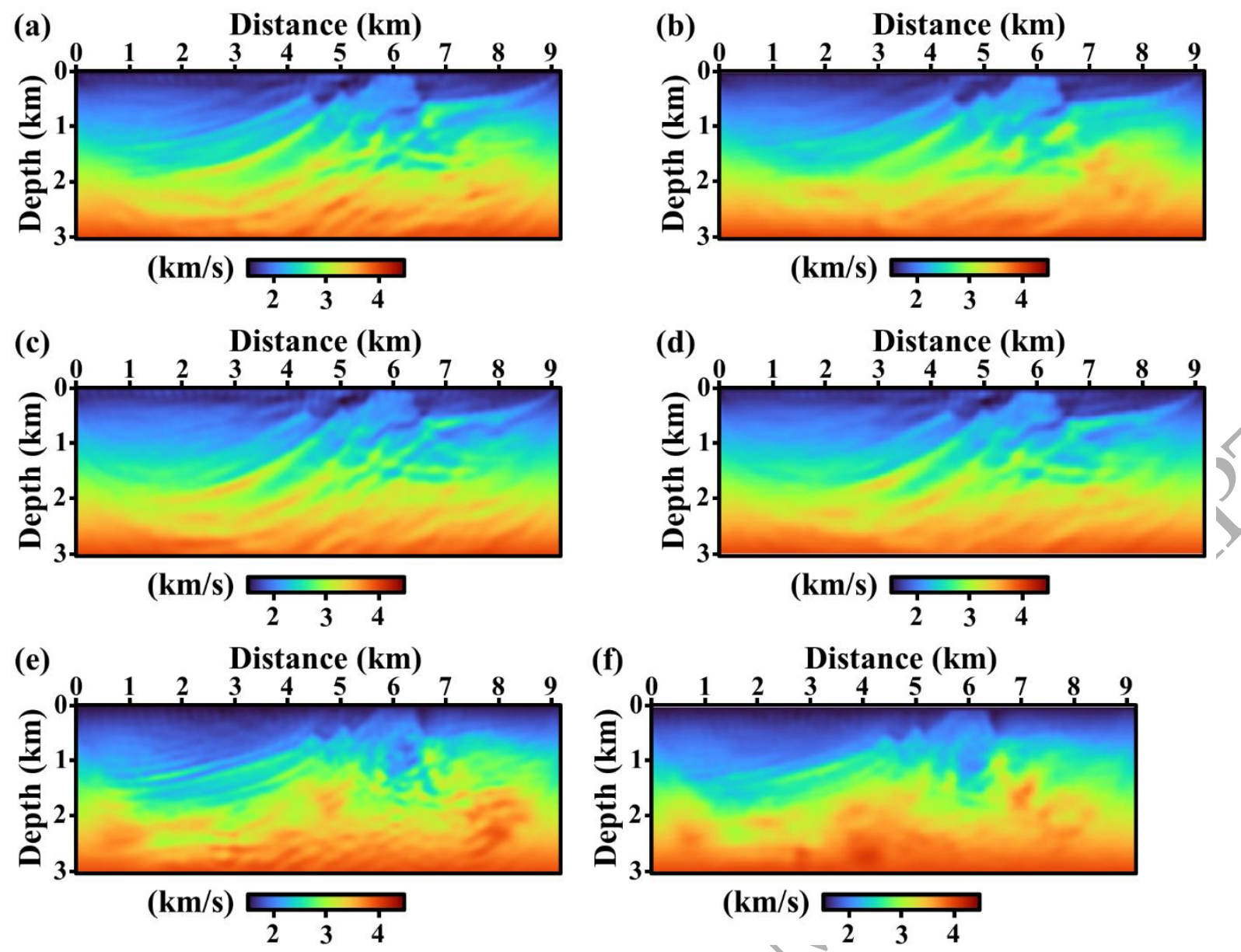

Fig. 8. The background velocity models obtained by two-step FWI using $(a, b)$ the full data, (c, d) the early arrival data and (e, f) the pre-critical reflection data. The left panels (a, c, e) are filtered by mode II of DAF and the right panels (b, d, e) are filtered by mode IV of DAF during two-step inversion. 


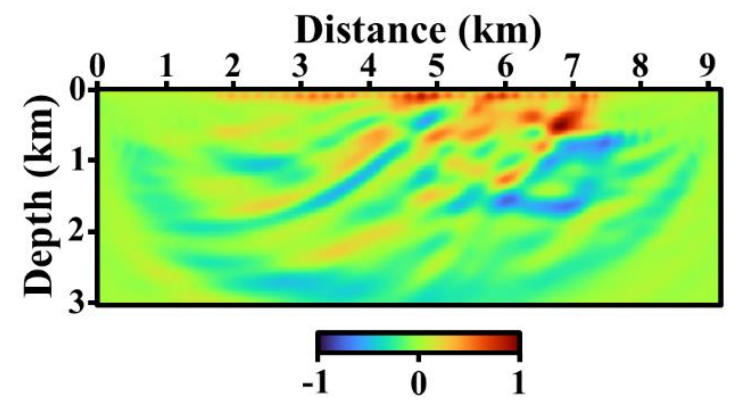

Fig. 9. The gradient obtained by applying mode IV of DAF at the first iteration of conventional FWI. 


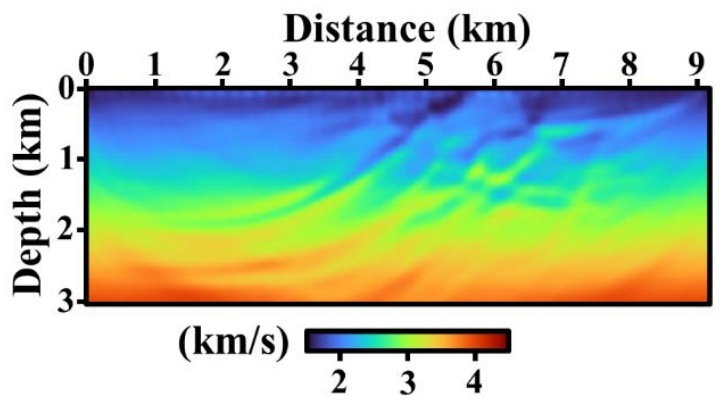

Fig. 10. The background velocity model obtained by applying mode IV of DAF in conventional FWI. 

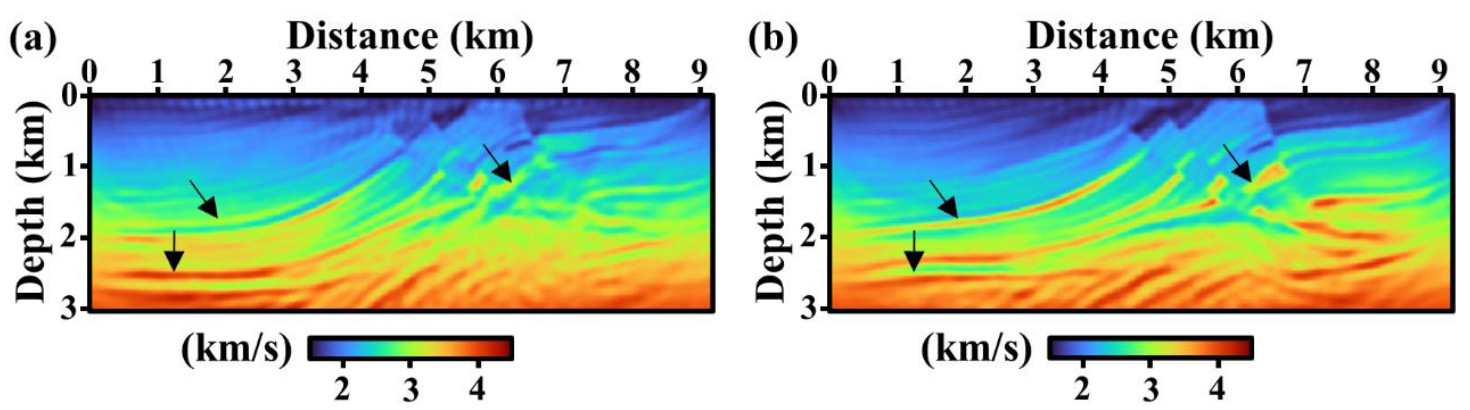

Fig. 11. Final inversion results obtained by subsequent FWI starting from (a) the linearlyincreasing velocity model and (b) the background velocity model obtained by two-step FWI. 

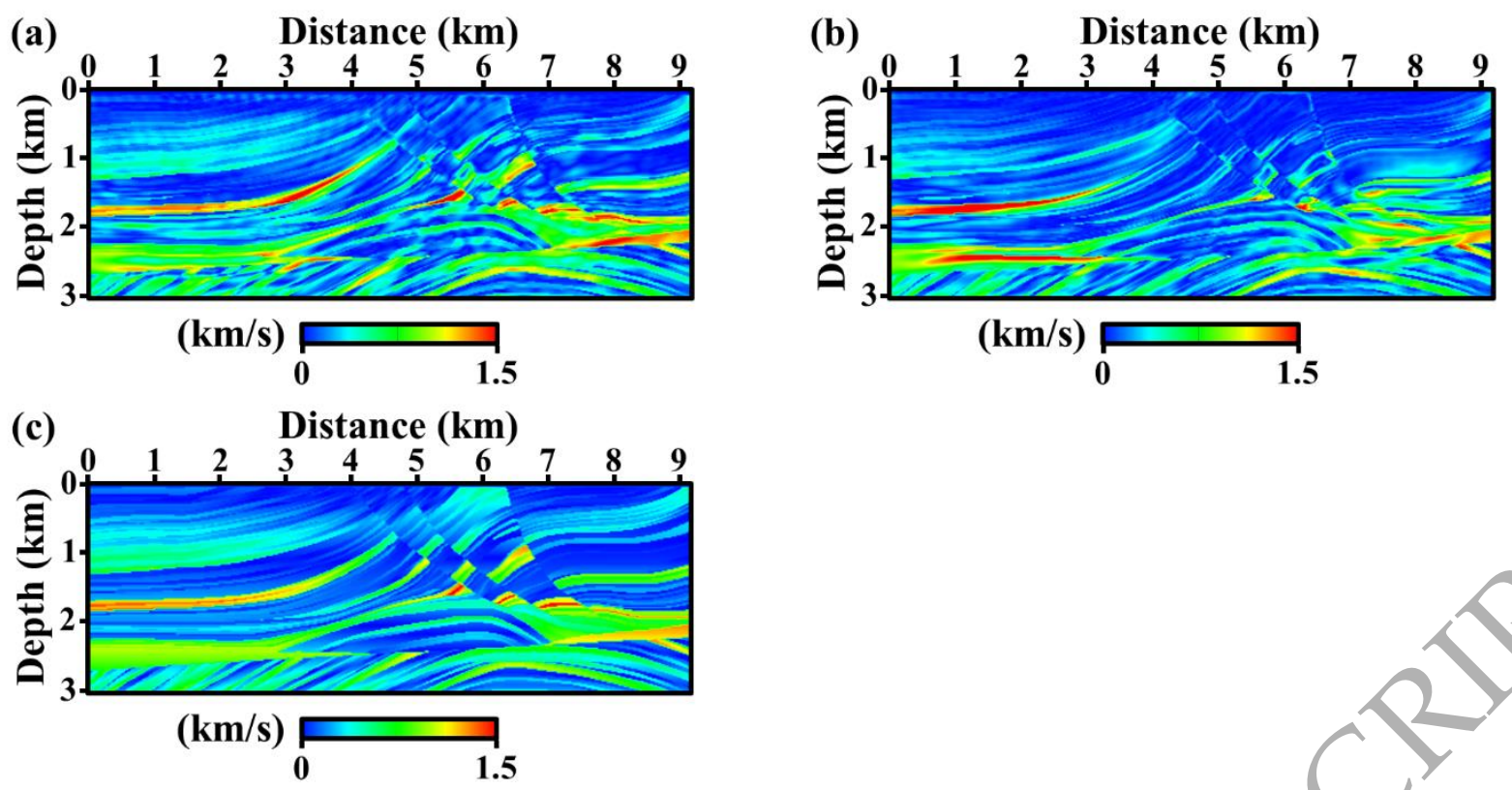

Fig. 12. Absolute differences between the true Marmousi-II velocity model and the final models inverted by subsequent FWI starting from (a) the linearly-increasing velocity model and (b) the background velocity model obtained by two-step FWI. For reference, the differences between the true and linearly-increasing velocity models are also displayed in (c) . 

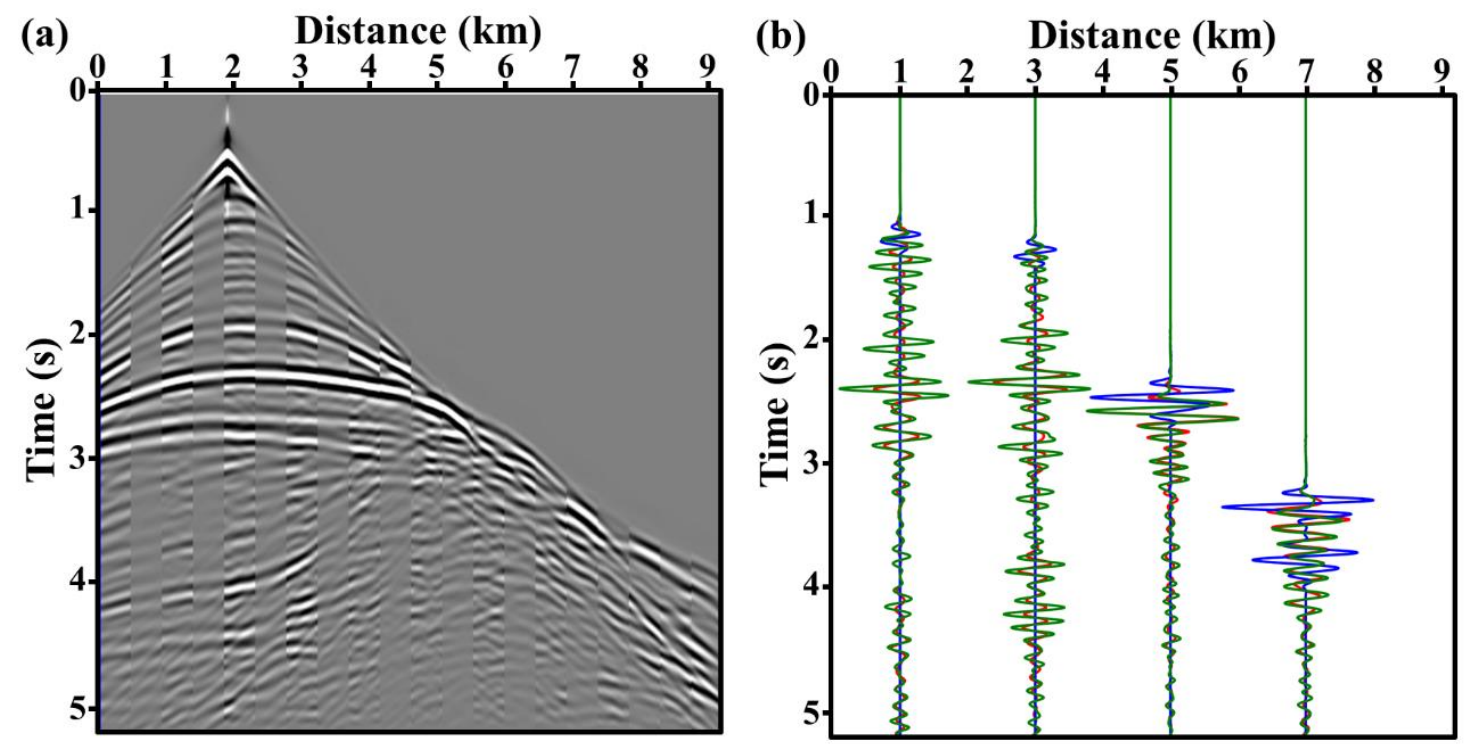

Fig. 13. (a) A representative shot gather at a distance of $1.9 \mathrm{~km}$ displayed by interweaving the observed data with the modelled data for the final inversion result (shown in Fig. 11b) every 23 traces. From left to right in the panel, the observed data are shown first followed by the modelled data. (b) The comparison of the traces of the observed (green) and modelled data for the linearly-increasing (blue) and final velocity models (red). 

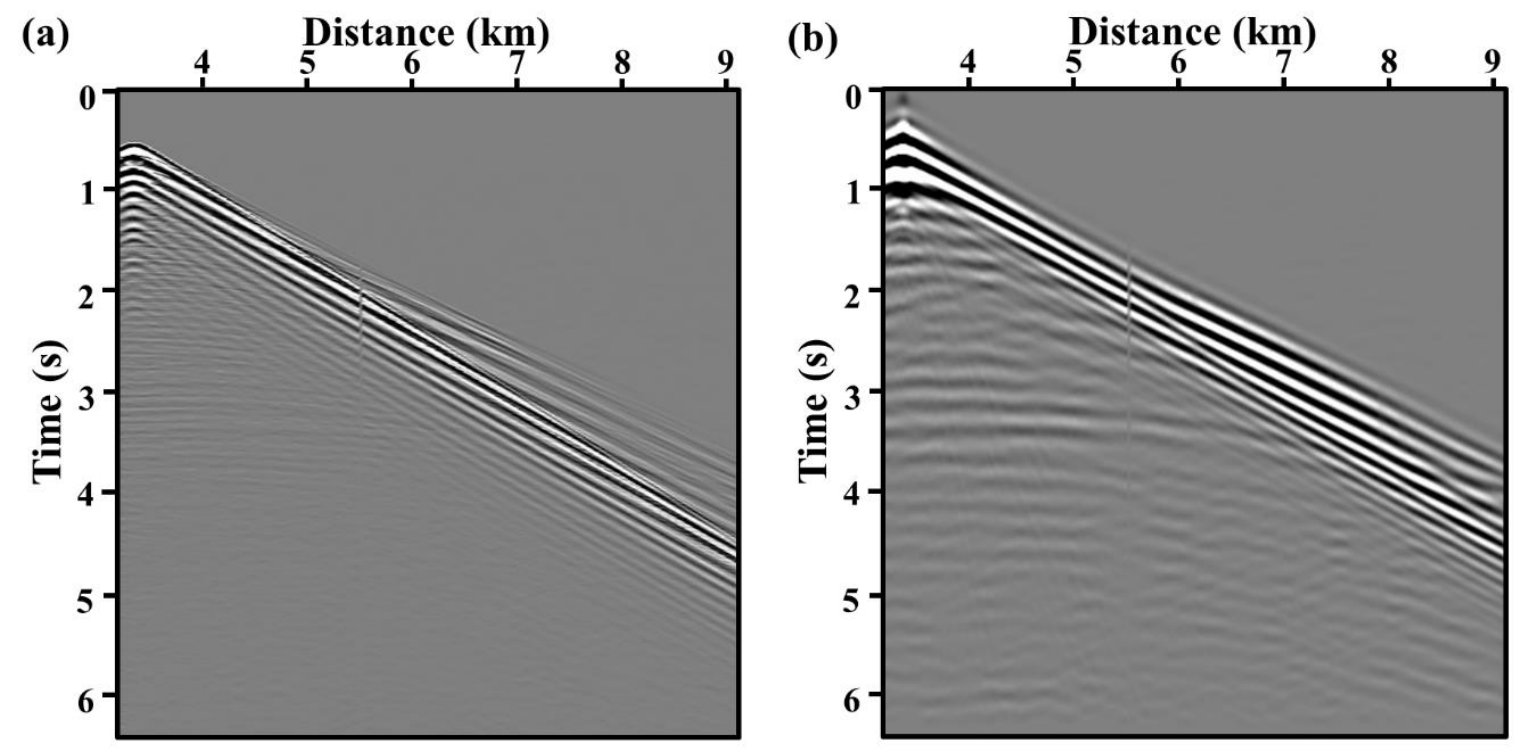

Fig. 14. (a) A representative shot gather of the Volve OBC data set at a distance of $3.35 \mathrm{~km}$ and (b) its filtered version obtained by the band-pass filter ranging from 2.5 to $5.6 \mathrm{~Hz}$. 

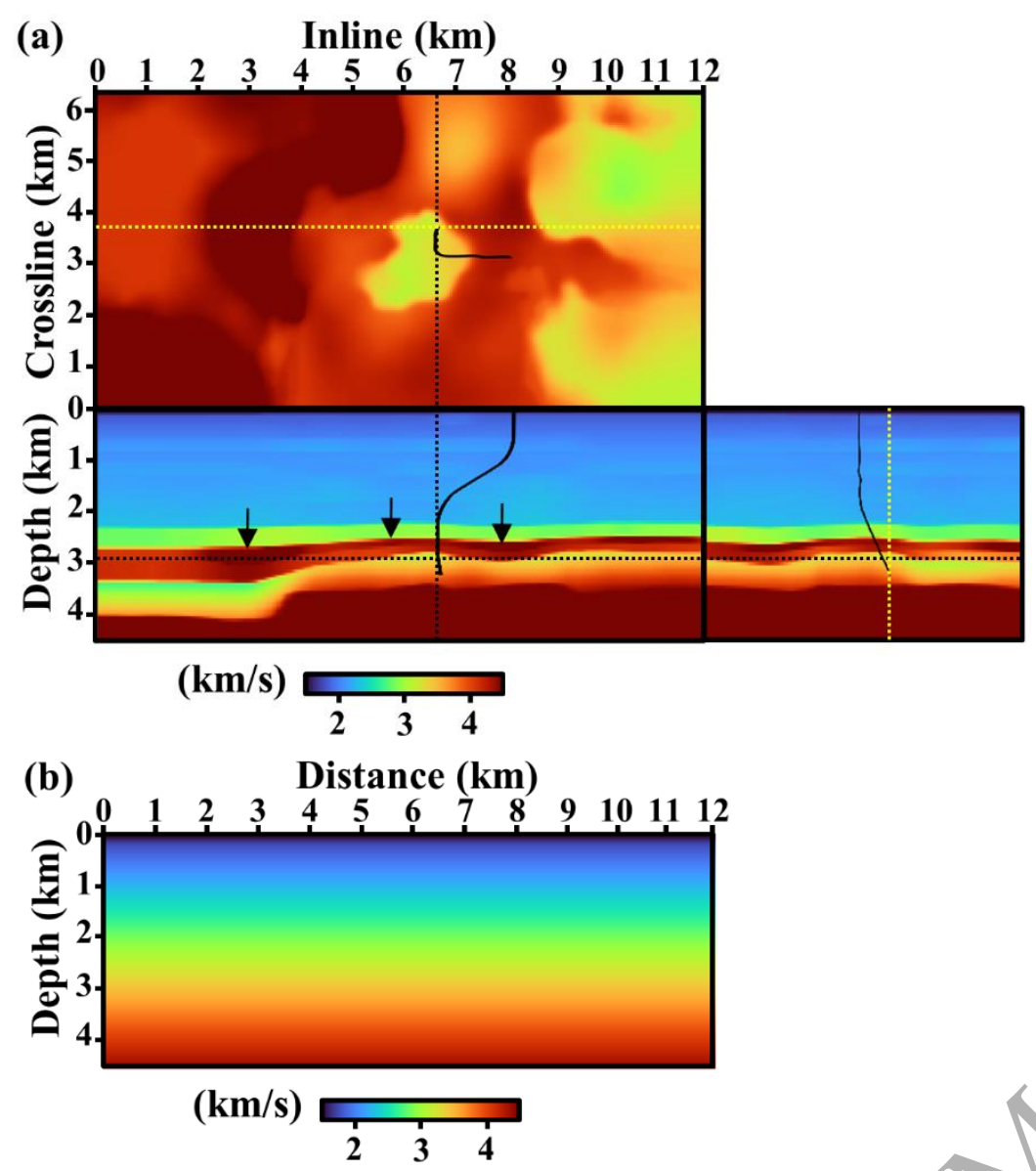

Fig. 15. (a) The 3D tomography $\mathrm{P}$-wave velocity model released along with the Volve data and (b) the linearly-increasing P-wave velocity model used as an initial guess. The yellow dashed lines indicate the section used for 2D acoustic FWI. The black solid lines indicate the path of the well. The black arrows indicate the top interface of the chalk layer in the tomography model. 


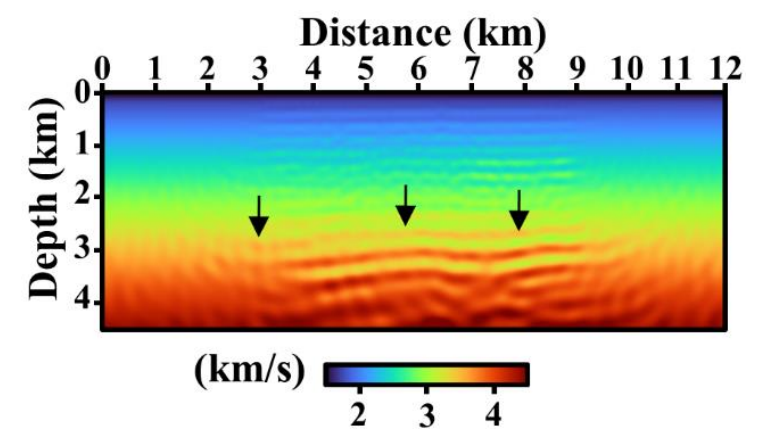

Fig. 16. The high-wavenumber velocity model $m_{0}+\delta m$ inverted using mode V of DAF in the inner loop of two-step FWI, when the background velocity model $m_{0}$ is assumed to be the linearly-increasing velocity model shown in Fig. 15(b). 


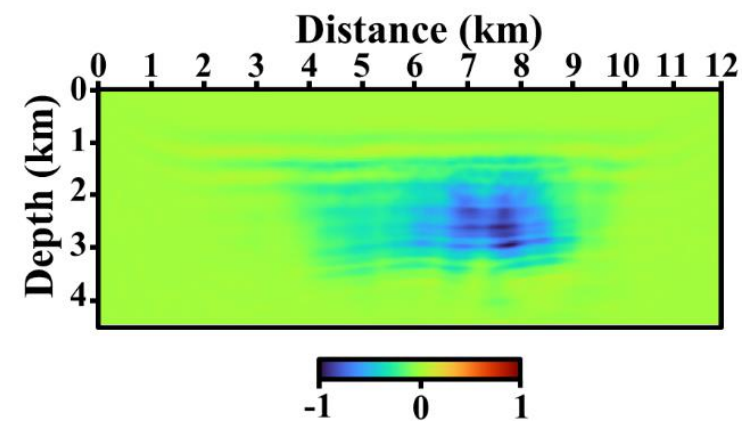

Fig. 17. The scaled gradient obtained at the first iteration of the outer loop of two-step FWI. 


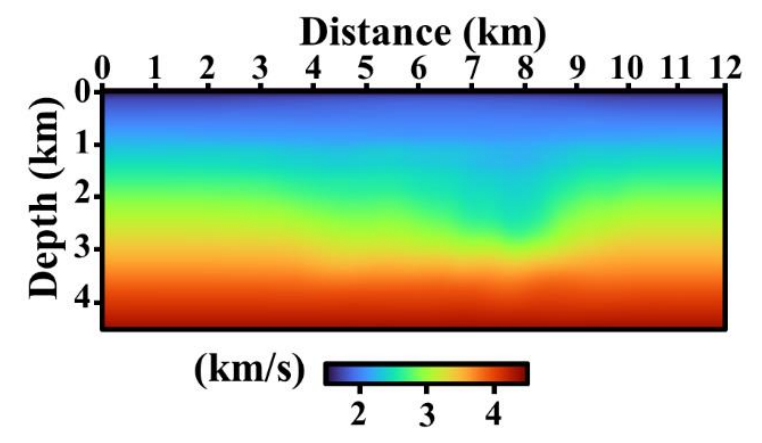

Fig. 18. The background velocity model obtained by two-step FWI. 


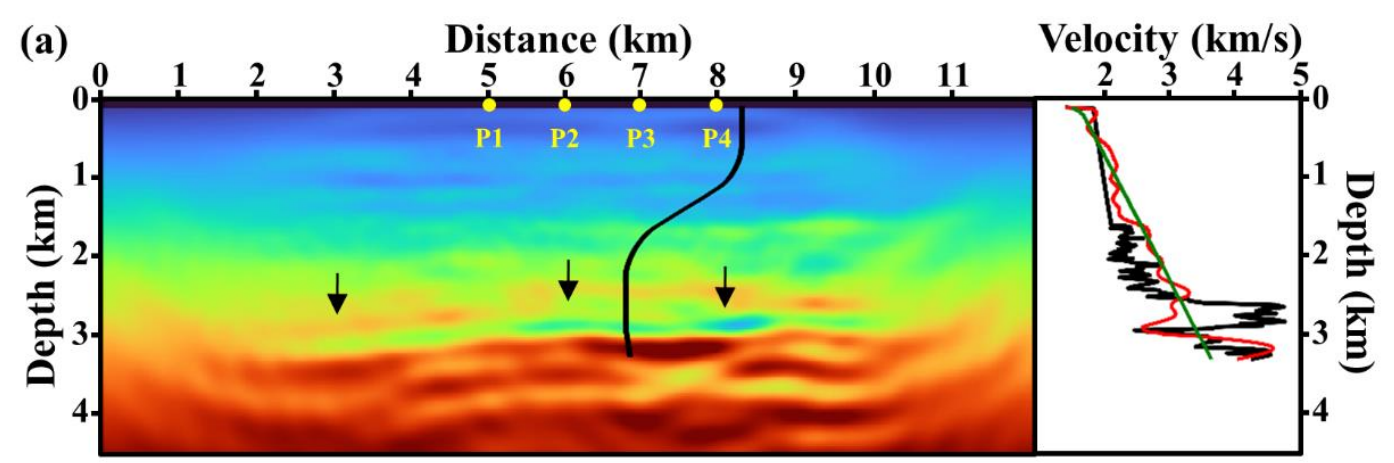

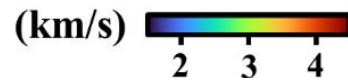

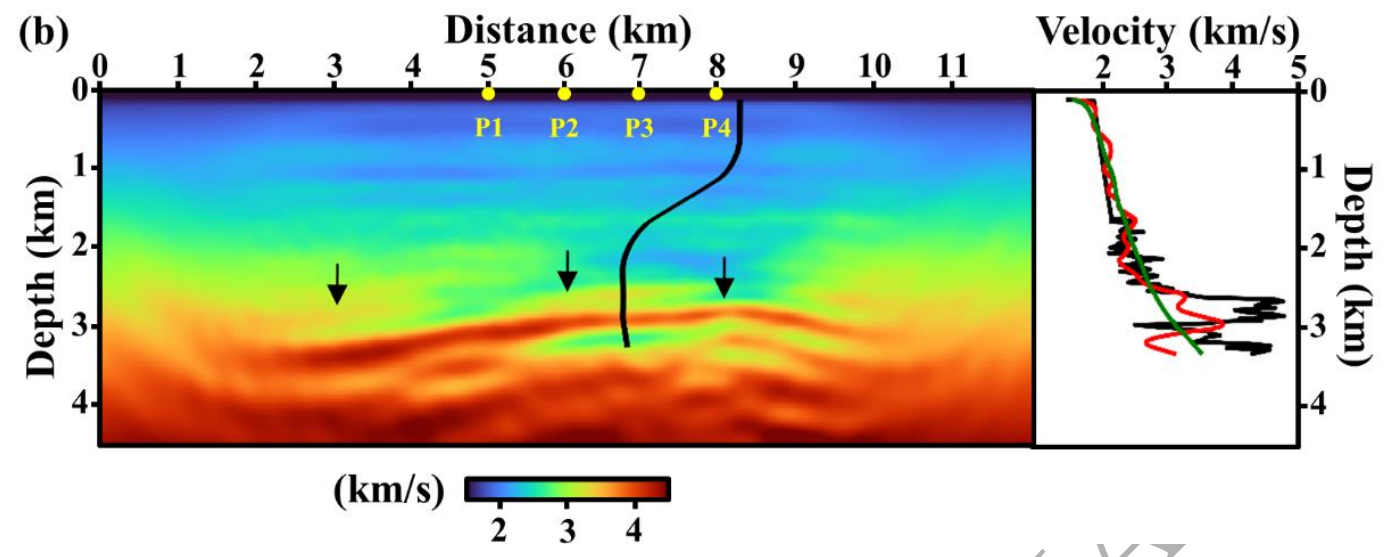

Fig. 19. Final inversion results and depth profiles along the well path obtained by subsequent FWI starting from (a) the linearly-increasing velocity model and (b) the background velocity model obtained by two-step FWI. In the right panels of (a) and (b), the depth profiles of starting (the green lines) and inverted velocity models (the red lines) are displayed with the well $\log$ (the black lines). The well log velocities above a depth of $1.6 \mathrm{~km}$ are not available and thus they are interpolated. The black lines in the left panels of (a) and (b) indicate the well path. The yellow dots indicate the locations for ADCIG in Fig. 20. 

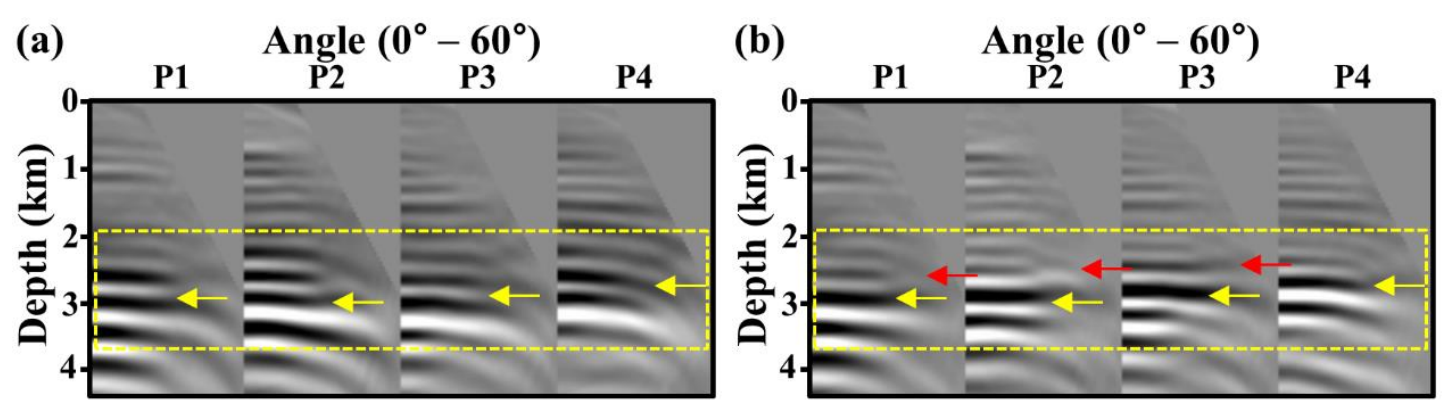

Fig. 20. ADCIGs for the final inversion results obtained starting from (a) the linearlyincreasing velocity model and (b) the background velocity model obtained by two-step FWI. The angle ranges from $0^{\circ}$ to $60^{\circ}$. Locations of $\mathrm{P} 1-\mathrm{P} 4$ are shown in Fig. 19. 


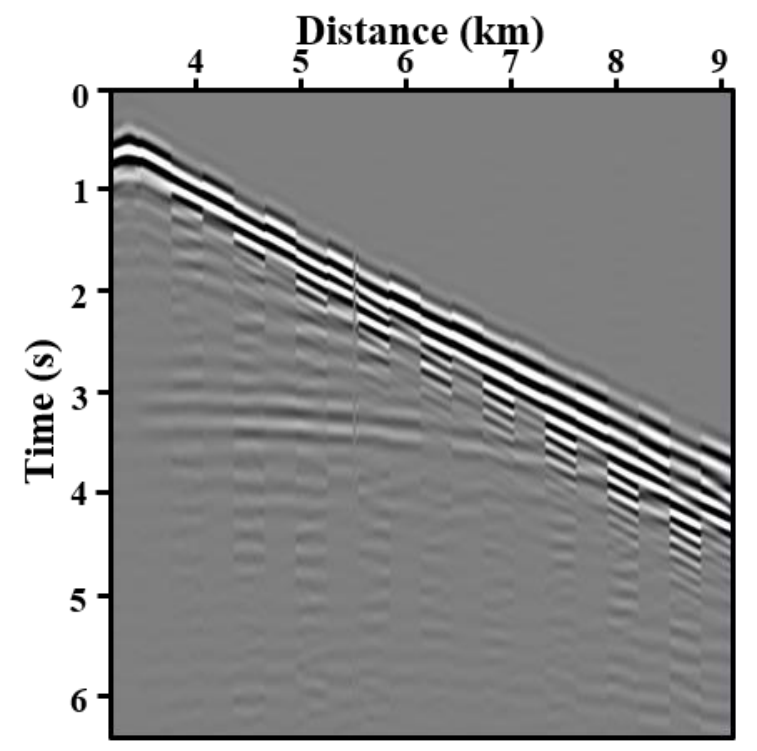

Fig. 21. A representative shot gather at a distance of $3.35 \mathrm{~km}$ displayed by interweaving the trace-by-trace-normalized Volve field data with the modelled data computed for the final inversion model (shown in Fig. 19b) every 12 traces. From left to right in the panel, the Volve field data are shown first followed by the modelled data. 